\title{
Notch1 regulates progenitor cell proliferation and differentiation during mouse yolk sac hematopoiesis
}

\author{
I Cortegano ${ }^{1,2,4}$, P Melgar-Rojas ${ }^{3,4,5}$, L Luna-Zurita ${ }^{3}$, M Siguero-Álvarez ${ }^{3}$, MAR Marcos $^{2, \text {, }}$, ML Gaspar ${ }^{1}$ and JL de la Pompa*,3
}

Loss-of-function studies have demonstrated the essential role of Notch in definitive embryonic mouse hematopoiesis. We report here the consequences of Notch gain-of-function in mouse embryo hematopoiesis, achieved by constitutive expression of Notch1 intracellular domain (N1ICD) in angiopoietin receptor tyrosine kinase receptor-2 (Tie2)-derived enhanced green fluorescence protein (EGFP ${ }^{+}$) hematovascular progenitors. At E9.5, N1ICD expression led to the absence of the dorsal aorta hematopoietic clusters and of definitive hematopoiesis. The EGFP ${ }^{+}$transient multipotent progenitors, purified from E9.5 to 10.5 Tie2-Cre; N1ICD yolk sac (YS) cells, had strongly reduced hematopoietic potential, whereas they had increased numbers of hemogenic endothelial cells. Late erythroid cell differentiation stages and mature myeloid cells $\left(\mathrm{Gr}^{+}{ }^{+}, \mathrm{MPO}^{+}\right)$were also strongly decreased. In contrast, EGFP ${ }^{+}$erythro-myeloid progenitors, immature and intermediate differentiation stages of YS erythroid and myeloid cell lineages, were expanded. Tie2-Cre;N1ICD YS had reduced numbers of CD41 ${ }^{++}$megakaryocytes, and these produced reduced below-normal numbers of immature colonies in vitro and their terminal differentiation was blocked. Cells from Tie2-Cre;N1ICD YS had a higher proliferation rate and lower apoptosis than wild-type (WT) YS cells. Quantitative gene expression analysis of FACS-purified EGFP ${ }^{+}$YS progenitors revealed upregulation of Notch1-related genes and alterations in genes involved in hematopoietic differentiation. These results represent the first in vivo evidence of a role for Notch signaling in YS transient definitive hematopoiesis. Our results show that constitutive Notch1 activation in Tie2 ${ }^{+}$cells hampers YS hematopoiesis of E9.5 embryos and demonstrate that Notch signaling regulates this process by balancing the proliferation and differentiation dynamics of lineage-restricted intermediate progenitors.

Cell Death and Differentiation (2014) 21, 1081-1094; doi:10.1038/cdd.2014.27; published online 28 February 2014

Notch is a highly conserved signaling pathway that regulates cell fate decisions in a variety of processes, including embryonic and adult hematopoiesis. ${ }^{1,2}$ Notch proteins and their ligands are transmembrane proteins and, upon ligand binding, the Notch intracellular domain (NICD) is released from the membrane by two consecutive proteolytic cleavage events and translocates to the nucleus. In the nucleus, NICD heterodimerizes with the transcriptional repressor CSL/RBPJK and converts it into a transcriptional activator. NICD/RBPJK target genes include those encoding basic helix-loop-helix transcription factors of the Hes and Hey families. ${ }^{1}$ Truncated versions of Notch containing only the NICD result in constitutive activation of the pathway. ${ }^{3-5}$ Milner et $a l^{6}{ }^{6}$ demonstrated that Notch1 is expressed on human $\mathrm{CD} 34^{+}$bone marrow (BM) hematopoietic progenitor cells. Some years before, the human $\mathrm{NOTCH} 1$ gene was found to be disrupted by the chromosomal translocation $\mathrm{t}(7 ; 9)(q 34 ; q 34.3)$, which yields truncated activated NOTCH1 (TAN-1) transcripts and results in T-cell acute lymphoblastic leukemia $(\mathrm{T}-\mathrm{ALL}) .^{7}$ Indeed, more than $50 \%$ of $\mathrm{T}$-ALL patients carry mutations leading to constitutive activation of $\mathrm{NOTCH} 1$ in $\mathrm{T}_{\text {cell. }}{ }^{8}$ It is now well known that NOTCH1 activation has critical roles at multiple stages of $\mathrm{T}$-cell development. $^{2}$

Notch signaling elements are expressed during embryonic and adult hematopoiesis. ${ }^{9-11}$ During embryogenesis, hematopoiesis takes place in two different sites, the yolk sac (YS) and the para-aortic splanchnopleura region (P-Sp). ${ }^{12-14}$ Primitive hematopoiesis begins in the mouse at E7.0-7.5 in the $\mathrm{YS}$ blood islands as a transient wave that involves the production of primitive nucleated erythrocytes and certain myeloid progenitor cells. ${ }^{15}$ Adult-like definitive hematopoietic

\footnotetext{
${ }^{1}$ Immunology Department, Centro Nacional de Microbiología, Instituto de Salud Carlos III, Ctra. Majadahonda-Pozuelo, km 2, 28220 Madrid, Spain; ${ }^{2}$ Centro de Biología Molecular, Consejo Superior de Investigaciones Científicas (CBM-CSIC), Campus de Cantoblanco, 28049 Madrid, Spain and ${ }^{3}$ Program of Cardiovascular Developmental Biology, Department of Cardiovascular Development and Repair, Centro Nacional de Investigaciones Cardiovasculares (CNIC), Melchor Fernández Almagro 3, 28029 Madrid, Spain

${ }^{*}$ Corresponding author: JL de la Pompa, Cardiovascular Developmental Biology, Centro Nacional de Investigaciones Cardiovasculares (CNIC), Melchor Fernández Almagro 3, 28029 Madrid, Spain. Tel: + 3491 4531334; Fax: +34 91 4531304; E-mail: jlpompa@cnic.es

${ }^{4}$ These authors contributed equally to this work.

${ }^{5}$ Current address: Laboratorio de Neurobiología Celular, Instituto de Investigación en Discapacidades Neurológicas, Universidad de Castilla-La Mancha, Almansa 14, 02006 Albacete, Spain.

Deceased.

Abbreviations: 7-AAD, 7-aminoactinomycin D; AGM, aorta-gonads-mesonephros; BM, bone marrow; DAPI, 4',6-diamidino-2-phenylindole; EdU, 5-ethinyl-2'deoxyuridine; EGFP, enhanced green fluorescence protein; FSC, forward scatter; HSC, hematopoietic stem cells; MFI, mean fluorescence intensity; MK, megakaryocyte; mAbs, monoclonal antibodies; N1ICD, Notch1 intracellular domain; P-Sp, para-aortic splanchnopleura region; PI, propidium iodide; qRT-PCR, quantitative real-time PCR; SSC, side scatter; Tie2, tyrosine kinase receptor-2; vWF, von Willebrand factor; WT, wild type; YS, yolk sac

Received 20.9.13; revised 27.12.13; accepted 28.1.14; Edited by R De Maria; published online 28.2.14
} 
cells comprising erythrocytes, macrophages and granulocytes are produced in the YS at E8.25 from transient multipotent progenitors that lack long-term reconstitution potential. ${ }^{16}$ At E9.5-10, the intraembryonic P-Sp develops into the aorta-gonads-mesonephros (AGM) region and produces hematopoietic stem cells (HSCs) with long-term reconstitution potential that give rise to all cells of the adult hematopoietic system. These intraembryonic HSCs appear in a cell-autonomous manner as cell clusters in the floor of the dorsal aorta and the vitelline and umbilical arteries. ${ }^{17-20} \mathrm{HSCs}$ first generate committed, transit-amplifying progenitors that then differentiate into hematopoietic lineage-restricted progenitor cells. ${ }^{21}$ HSCs and progenitor cells migrate to colonize the fetal liver, the spleen, the thymus and finally the BM, which is the main hematopoietic organ during adult life. ${ }^{21}$

Normal hematopoiesis is the result of a highly regulated balance between self-renewal, proliferation and differentiation of stem and progenitor cells. Numerous reports based on in vitro and in vivo experiments strongly support a role for Notch in the self-renewal of hematopoietic stem and progenitor cells, and alterations to the Notch pathway disrupt hematopoietic differentiation. ${ }^{22-25}$ Targeted inactivation of the Notch signaling components Notch1, RPBJk, Jag1 and Mib1 showed that Notch is essential for definitive hematopoiesis in the intraembryonic P-Sp/AGM region. ${ }^{26,27}$ The tyrosine kinase receptor-2 (Tie2) is expressed on vascular endothelium and on HSCs, and $\mathrm{Tie}^{+}{ }^{+}$cells contain hemangioblasts able to differentiate into hematopoietic and endothelial lineages. ${ }^{28}$ Since both Tie2 and Notch1 intracellular domain (N1ICD) proteins have similar expression patterns very early in the YS blood island, ${ }^{29,30}$ we have used the Tie2-Cre driver line ${ }^{31}$ to overexpress Notch1 (N1ICD-EGFP (enhanced green fluorescence protein)). ${ }^{32}$ In this report, we show that constitutive Notch1 activation in $\mathrm{Tie}^{+}$cells impairs definitive hematopoiesis in the E9.5 embryo and produces severe alterations in YS transient definitive and primitive hematopoiesis. These results demonstrate that Notch signaling has an important function in YS-derived hematopoiesis by balancing the dynamics of proliferation and differentiation of lineagerestricted intermediate progenitors.

\section{Results}

Constitutive N1ICD expression in $\mathrm{Tie}^{+}$progenitors impairs definitive intraembryonic hematopoiesis. To study the consequences of Notch1 gain-of-function in hematopoiesis, we used the conditional N1ICD transgenic line $R 26 N 1 / C D$, which upon Cre activation expresses the N1ICD (amino acids 1749-2293) and nuclear-localized enhanced GFP. ${ }^{32}$ Tie2-Cre;N1ICD embryos that constitutively expressed N1ICD and EGFP in Tie2 ${ }^{+}$hematovascular progenitor cells died at E11.0. ${ }^{33}$ At E9.5 (Figures 1a-d), transgenic embryos were smaller than wild-type (WT) littermates and the YS was pale and lacked well-formed blood vessels (Figure 1b). Similarly, although the dorsal aorta and the umbilical and vitelline arteries were preserved, the intraembryonic P-Sp/AGM area lacked hemoglobinized cells (Figure 1d). In most Tie2-Cre;N1ICD embryos, the YS contained small, randomly located concentrations of hemoglobinized red blood cells (Figure 1b). This phenotype
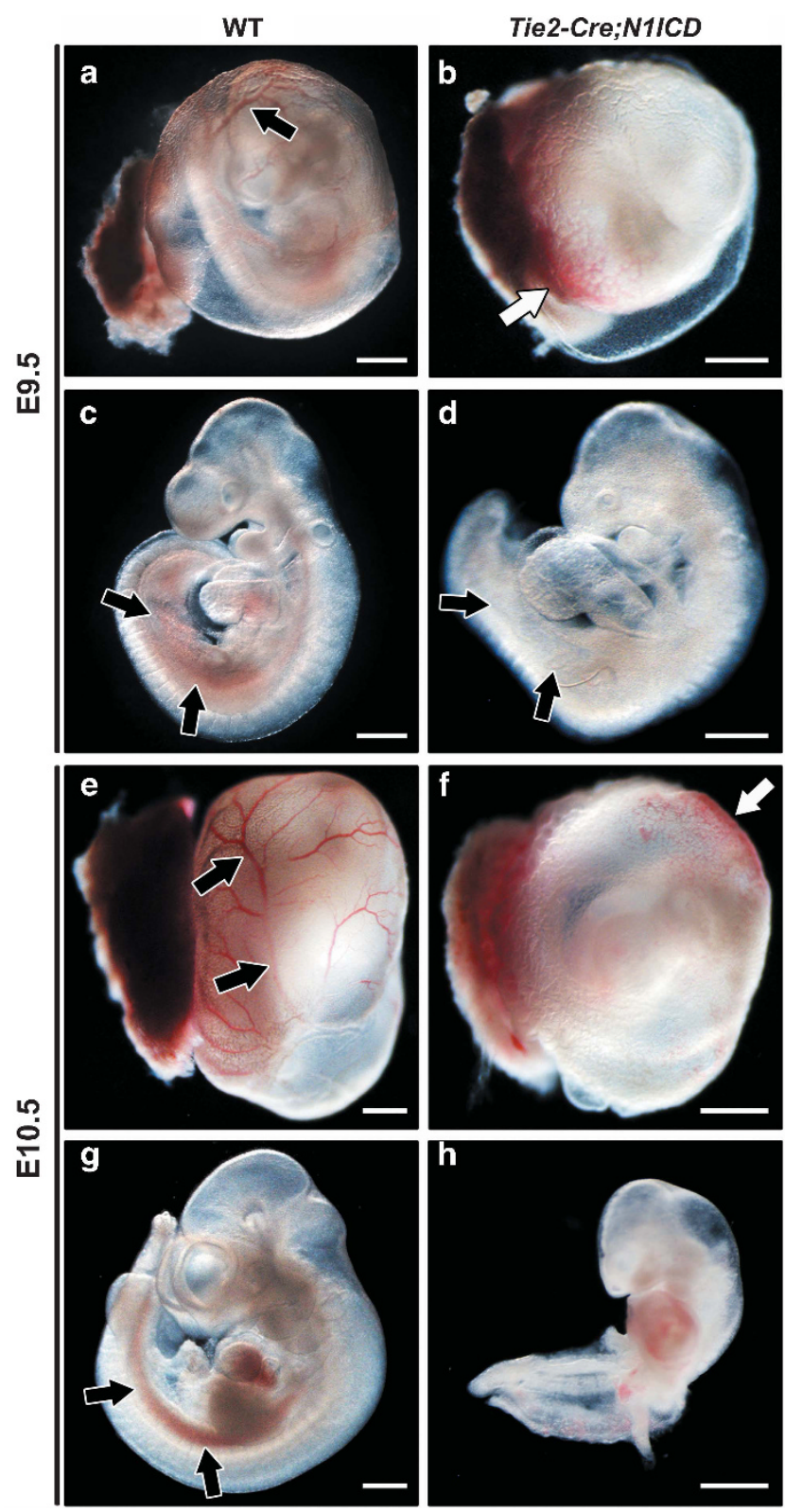

Figure 1 Constitutive Notch1 activation on $\mathrm{Tie}^{+}$progenitor cells impairs hematopoietic development. WT and Tie2-Cre;N1ICD embryos (left and right panels, respectively) at E9.5 (a-d) and E10.5 (e-h), with the YS (a, b, e and f) and without it (c, $\mathbf{d}, \mathbf{g}$ and $\mathbf{h})$. (a, b) Morphology of the YS of WT and Tie2-Cre;N1/CD embryos. WT YS contains normally developed blood vessels (black arrow in a) that are absent in transgenic YS. Tie2-Cre;N1ICD YS is very pale, with hemoglobinized cells concentrated at one pole (white arrow in $\mathbf{b}$ ). (c, d) Lateral views of WT and Tie2-Cre;N1/CD embryos. Black arrows indicate the P-Sp/AGM region with hemoglobinized content in WT, and the pale region in the transgenic embryo, indicative of defective definitive hematopoietic development. (e-h) At E10.5, defects in Tie2-Cre;N1ICD YS and embryos are more severe. Black arrows in (e) mark the well-developed WT YS blood vessels that are never seen in transgenic YS $(f)$, which instead contains randomly located pockets of high hemoglobin content (white arrow). Lateral views of WT (g) and Tie2-Cre;N1/CD (h) embryos show the hemoglobinized dorsal aorta in the AGM region (black arrows) of a WT embryo that is absent in the transgenic embryo. Scale bars: $800 \mu \mathrm{m}$

suggested a severe alteration in embryonic angiogenesis associated with defects in primitive or definitive hematopoiesis. The phenotype worsened by E10.5 (Figures 1e-h) and was 
accompanied by severe cardiac defects that presumably resulted in hemodynamic alterations, causing embryonic death. $^{33}$

CD31/PECAM1 was expressed on hematopoietic and endothelial cells in the P-Sp/AGM dorsal aorta and the YS vitelline artery (Supplementary Figure $1 \mathrm{a}-\mathrm{j}$ ), indicating that early endothelial differentiation occurs in these vessels. Nevertheless, the arterial marker Efnb2 was ectopically expressed in venous endothelium (Supplementary Figure $1 \mathrm{k}$ and I) while the venous marker Ephb4 was not expressed (Supplementary Figure $1 \mathrm{~m}$ and $\mathrm{n}$ ). N1ICD staining labels arterial endothelium (Supplementary Figure 10 and Del Monte et al. $^{30}$ ), so the observation that Tie2-Cre-driven ectopic N1ICD expression promotes arterial differentiation of venous endothelium is consistent with the requirement of Notch in arterial endothelium differentiation. ${ }^{34}$ LacZ staining of Tie2-Cre;R26R embryos (Supplementary Figure 1p) indicates that this driver is active throughout the endothelium and circulating cells and is an excellent tool for studying the effect of Notch overexpression in these tissues.

Histological analysis of embryos and YS revealed that, compared with the WT extraembryonic vasculature formed by the capillary plexus (Figure 2a) and vessels full of circulating cells (Figure 2c), extraembryonic vessels in Tie2-Cre;N1ICD embryos were dilated and expanded (Figure 2b). Moreover, transgenic embryos exhibited two types of blood islands: some with the lumen almost empty (Figure 2d) and others full of cells (Figure $2 b$ ). These presumably corresponded to the hemoglobinized YS pockets described above (Figures $1 \mathrm{~b}$ and $\mathrm{f}$ ).

Confocal analysis of WT (Figure $2 \mathrm{c}^{\prime}$ ) and transgenic YS showed that EGFP expression labeled most although not all circulating cells within the vessels, indicating that Tie2-Credriven ectopic N1ICD expression was not uniform (Figure 2d'). At E9.5, the Tie2-Cre;N1ICD dorsal aorta was still a bilateral artery (Figure 2f) indicating a delay in vascular development. Unlike in the WT situation, where hematopoietic cell clusters were observed budding from the ventral wall of the dorsal aorta (Figure 2e), no clusters were detected in

Figure 2 Tie2-Cre;N1ICD embryos display severe vascular alterations and a blockade of intraembryonic definitive hematopoiesis. (a-d) Bright-field images of H\&E-stained transverse sections of YS from E9.5 WT (a, c) and Tie2-Cre;N1ICD (b, d) embryos, showing the differences in blood vessel diameter and circulating cell number. Transgenic YS (b) exhibits two types of blood island, with the lumen empty (black arrowhead) or with a high concentration of circulating cells (white arrowhead). Panels (c) and (d) show detailed views of a normal blood vessel in WT YS (c) and a dilated vessel of Tie2-Cre;N1ICD YS (d). ( $\left.\mathbf{c}^{\prime}, \mathbf{d}^{\prime}\right)$ Confocal image of a WT $\left(\mathbf{c}^{\prime}\right)$ and Tie2-Cre;N1/CD YS ( $\left.\mathbf{d}^{\prime}\right)$ stained with DAPI. The white arrowheads in point to the circulating cells in WT YS that do not express EGFP $\left(\mathbf{c}^{\prime}\right)$, and to EGFP ${ }^{+}$coexisting with EGFP - circulating cells in the transgenic YS $\left(\mathbf{d}^{\prime}\right)$. (e, f) Transverse sections of E9.5 dorsal aorta at the P-Sp/AGM region level in WT (e) and Tie2-Cre;N1/CD embryos (f); transgenic animals have bilateral dorsal aortas (white arrows) and lack the hematopoietic cell clusters that start to bud off from the endothelium in WT embryos (black arrows in e). G, gut. (g, h) In situ hybridization of $A M L 1 /$ Runx 1 in WT (g) and Tie2-Cre;N1ICD (h) vitelline artery. Hematopoietic cells almost obliterate the lumen of WT vitelline artery, whereas a single small hematopoietic cell cluster is seen in the Tie2-Cre;N1ICD vessel (black arrow in h). (i) Dot plots showing a marked reduction in the $\mathrm{CD}_{4} 5^{+}$cell population in the caudal portion of Tie2-Cre;N1/CD (right) versus WT (left) embryos. Data show frequency (mean \pm S.E.M., $N=3$ ). Scale bars: $25 \mu \mathrm{m}$
Tie2-Cre;N1/CD dorsal aorta (Figure 2f) or umbilical and vitelline arteries (data not shown). Nevertheless, the vitelline artery of $10 \%$ of the embryos contained cell clusters populated by hematopoietic progenitor cells, demonstrated by the normal expression of $A M L 1 /$ Runx 1 mRNA (Figures $2 \mathrm{~g}$ and $\mathrm{h}$ ).

These results suggest that constitutive Notch1 activation promotes an early blockade in the generation of definitive hematopoietic cell clusters. Supporting this, flow cytometry analysis detected 8.4-fold fewer circulating EGFP ${ }^{+} \mathrm{CD}_{4}{ }^{+}$ cells in E9.5 Tie2-Cre;N1ICD embryos (Supplementary

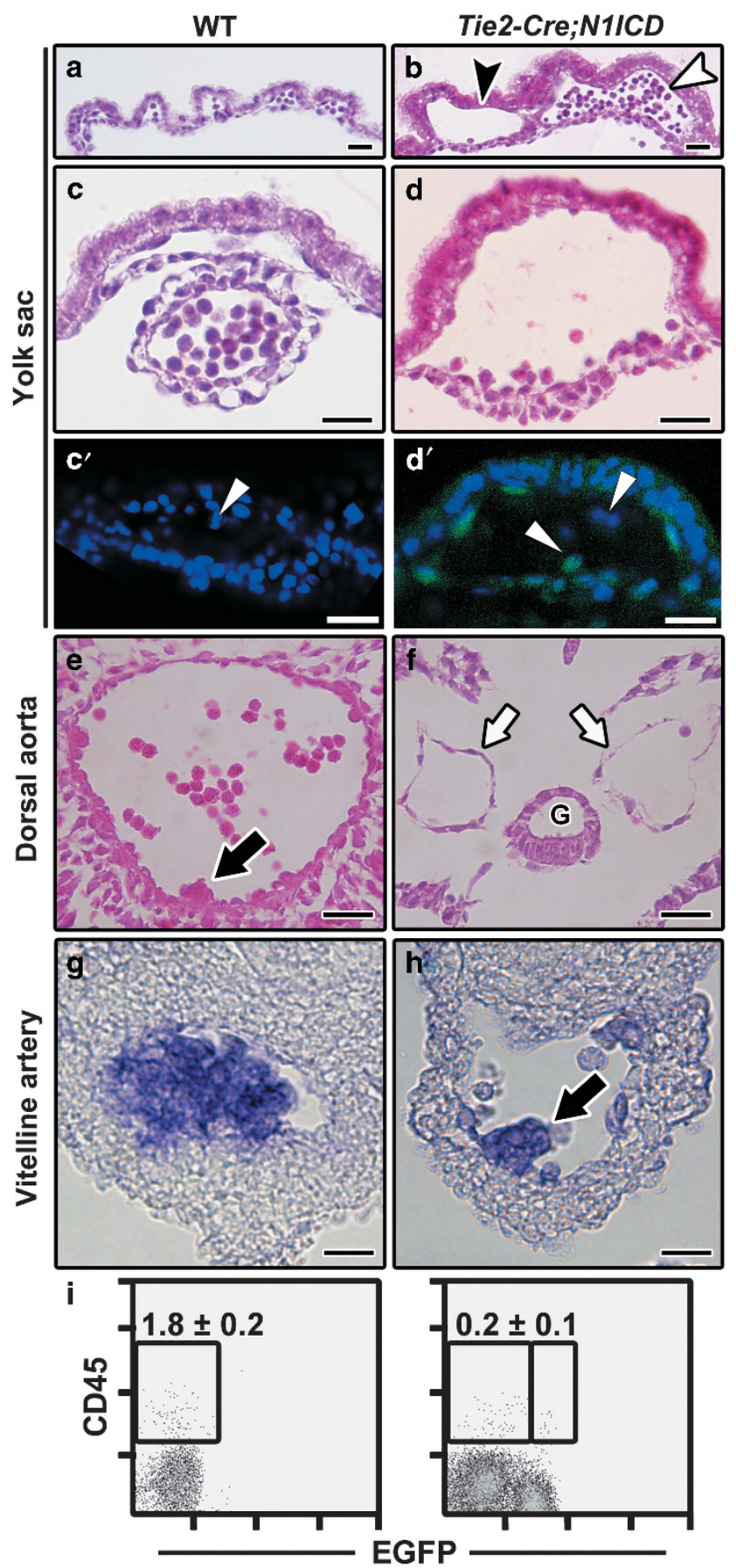


Figure 2a). In the same embryos, the intraembryonic CD45 ${ }^{+}$ population was almost absent in the caudal region (which includes the P-Sp/AGM) (Figure 2i). These data suggest that constitutive Notch1 expression in $\mathrm{Tie}^{+}{ }^{+}$cells leads to defects in YS hematopoietic and vascular development and to a blockade of intraembryonic definitive hematopoiesis similar to that described for Notch-deficient mice. ${ }^{26,35-37}$

\section{N1ICD expression reduces the hematopoietic potential and favors the endothelial phenotype of $\mathrm{Tie}^{+}$cells in} the YS. Flow cytometry analysis of Tie2-Cre;N1ICD YS cells at E9.5 showed that just $40-60 \%$ of the recovered cells were $\mathrm{EGFP}^{+}$, which may suggest incomplete Cre-mediated recombination and activation of the EGFP transgene (Figure 3a). The number of $\mathrm{EGFP}^{+}$and $\mathrm{EGFP}^{-}$cells recovered from YS of Tie2-Cre;N1ICD mice was lower than that recovered from WT mice (Figure 3b; Supplementary Figure 2b). The co-expression of EGFP and N1ICD in Tie2Cre; N1/CD mice ${ }^{33}$ allowed us to examine the hematopoietic potential of FACS-purified total EGFP ${ }^{+}$YS cell populations and of EGFP ${ }^{+}$cells expressing the stem cell factor (SCF) receptor (c-Kit) at E9.5. After 7 days in methylcellulose cultures, the numbers of colonies derived from E9.5 EGFP ${ }^{+}$ cells, both total and c-Kit positive, was 10- to 20-fold lower than those derived from WT cells (Figure 3c); however, numbers of primitive erythroid colonies were normal at E8.5 in total YS EGFP ${ }^{+}$and WT cells (Supplementary Figure $2 \mathrm{c}$ ). In addition, whereas colonies growing from E9.5 WT and EGFP $^{-}$cultures were BFU-E and CFU-M (Figure 3d, left panel and not shown), the small number of colonies detected in $\mathrm{EGFP}^{+}$cultures contained elongated fibroblast-like cells surrounding central clusters of small round cells (Figure 3d, right panel). When $\mathrm{EGFP}^{+}$colonies were transferred to fibronectin-coated plates, tube-like structures developed after $48 \mathrm{~h}$ that appeared to be pseudo-endothelial cords (Figure $3 e$ ). These cells were positive for the endothelialspecific marker von Willebrand factor (vWF) (Figure 3f). In agreement with these results, the $\mathrm{CD} 31^{+} \mathrm{CD} 41^{-} \mathrm{CD} 45^{-}$ Mac1 ${ }^{-}$TER $119^{-}$hemogenic endothelial cells ${ }^{38}$ were significantly increased in EGFP ${ }^{+}$cells from Tie2-Cre;N1ICD YS overexpressing Notch1 at E9.5 (Figures $3 \mathrm{~g}$ and $\mathrm{h}$ ).

In the WT YS, two cell populations could be distinguished ex vivo according to the levels of $\mathrm{c}-\mathrm{Kit}, \mathrm{C}-\mathrm{Kit}^{++}$cells, corresponding to immature progenitor cells, and c-Kit ${ }^{+}$cells, associated with more mature cellular stages. ${ }^{39}$ The relative size of the ${\mathrm{c}-\mathrm{Kit}^{+}}^{+}$population was larger in $\mathrm{YS}$ cells from $\mathrm{EGFP}^{+}$Tie2-Cre;N1ICD embryos (Figures 3i and j), whereas that of the $\mathrm{C}-\mathrm{Kit}^{+}{ }^{+}$cells was similar. Interestingly, some of

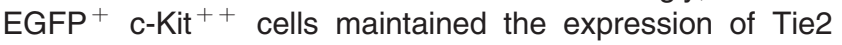
whereas WT c-Kit ${ }^{++}$cells and EGFP ${ }^{-}$cells did at very low levels, and more than half of them did not express CD31 or KDR (Supplementary Figure $3 a$ and b). In absolute terms, $\mathrm{EGFP}^{+}$cell suspensions contained 1.5 -fold more $\mathrm{c}-\mathrm{Kit}^{+}$cells than WT cell suspensions, whereas immature $\mathrm{c}-\mathrm{Kit}^{+}{ }^{+}$cells were in fact less abundant in Tie2-Cre;N1ICD mice (0.6-fold lower than WT). EGFP ${ }^{-}$cells in Tie2-Cre;N1ICD YS (Figure $3 a$ ) could in principle serve as an internal control for the effects of Notch1. However, both the numbers of EGFP ${ }^{-}$ ${\mathrm{c}-\mathrm{Kit}^{+}}^{+} \mathrm{I}^{++}$cells and the CFUs obtained from them (Figures $3 \mathrm{c}$ and j) were markedly reduced with respect to cells and colonies derived from WT embryos, indicating that these EGFP $^{-}$cells were affected by intracellular Notch1 expression in EGFP ${ }^{+}$cells.

Erythroid and megakaryocyte development is blocked in Tie2-Cre;N1ICD mice. Pellets obtained by centrifugation of WT YS were reddish, whereas those from Tie2-Cre;N1ICD YS were pale (Figure 4a), suggestive of an abnormal erythropoiesis. Immunostaining of YS cells for c-Kit, CD71 and TER119 allows identification of several differentiation stages in the erythroid developmental pathway. The proerythroblast stage in mouse BM includes large cells that co-express c-Kit, CD71 and low levels of the erythroid-specific marker TER119. ${ }^{40}$ Analysis of the expression of CD71 and TER119 in WT and EGFP ${ }^{+}$cells at E9.5 revealed markedly lower numbers of basophilic CD71 ${ }^{++}$TER $119^{++}$erythroblasts in Tie2-Cre;N1ICD mice (R2), accompanied by an accumulation of less mature CD71 ${ }^{++}$TER $119^{-1+}$ proerythroblasts (R1) (Figure 4b). This block in the differentiation of $\mathrm{TER}_{119^{+}}$cells was observed already at E8.5 (Supplementary Figure 2d), indicating that Notch1 overexpression affects also the primitive erythropoiesis wave. Cytospin preparations from total WT and EGFP ${ }^{+}$Tie2Cre; N1ICD cells showed that N1ICD-expressing cells had a high nucleocytoplasmic ratio and a more acidophilic cytoplasm (Figure 4c). Among the EGFP ${ }^{+}$cells we observed an immature $\mathrm{c}-\mathrm{Kit}^{+}$TER $119^{+}$population not seen in WT cells (Figure $4 d$ ), showing that part of the ${\mathrm{C}-\mathrm{Kit}^{+}}^{+}$population

Figure 3 Immature progenitor cell populations in E9.5 Tie2-Cre;N1/CD mice have a weak hematopoietic potential and maintain endothelial progenitors. (a) Contribution of $\mathrm{EGFP}^{-}$and EGFP ${ }^{+}$(N1ICD transgene-expressing) cells to YS, blood and P-Sp in E9.5Tie2-Cre;N1ICD embryos. The graph shows the relative percentage of EGFP ${ }^{+}$and EGFP $^{-}$cells in preparations of the indicated cell suspensions (mean \pm S.E.M., $N=13$ for YS and P-Sp and $N=7$ for blood). (b) N1ICD expression in Tie2-Cre;N1/CD YS reduces the numbers of cells expressing and not expressing the transgene at E9.5. Bar chart shows quantification of YS cells in WT and in Tie2-Cre;N1/CD EGFP- and EGFP $^{+}$cell populations. Bars represent mean \pm S.E.M. $(N=15)$. (c) Hematopoietic potential of WT and Tie2-Cre;N1/CD YS cells. Cells were cultured in MethoCult3434 medium for 7 days. Graphs show the absolute numbers of BFU-E and CFU-M (erythroid and myeloid, respectively) per 100 cells, detected in cultures of total cells at E9.5 and E10.5 (left) or from FACS-purified c-Kit-expressing progenitor cells of WT and EGFP ${ }^{-}$and EGFP ${ }^{+}$Tie2-Cre;N1/CD cells at E9.5 (right). Data are mean \pm S.E.M. (N=3). (d) Microphotographs showing representative colonies of WT and EGFP ${ }^{+}$cells. The periphery of EGFP ${ }^{+}$colonies is populated by elongated, polarized cells. (e) Pseudoendothelial structures formed after in vitro culture of EGFP ${ }^{+}$CFUs on fibronectin-coated plates in the presence of the pro-endothelial factors bFGF and VEGF. (f) Merged microphotographs of EGFP ${ }^{+}$CFU cells stained with vWF antibody (left) or with an isotype control antibody (right). (g) Representative cytometry plots of E9.5 YS cells from WT and Tie2-Cre;N1/CD mice stained for CD41, CD31, CD45, TER119 and CD11b/Mac1. CD41 ${ }^{+} \mathrm{CD} 45^{+}$TER119 ${ }^{+} \mathrm{CD} 11 \mathrm{~b}^{+}$cells were electronically excluded, and CD31 ${ }^{+}$is plotted showing green channel fluorescence to identify EGFP ${ }^{+}$cells. Boxed areas define hemogenic EC cells as CD $31^{+} \mathrm{CD}_{41} 1^{-} \mathrm{CD} 45^{-} \mathrm{TER}_{119^{-} \mathrm{CD} 11 \mathrm{~b}}{ }^{-}$. Numbers are percentages of gated cells, as mean \pm S.E.M, $(N=4)$. (i) Representative cytometry plots of E9.5 YS cells from WT and Tie2-Cre;N1/CD mice stained for c-Kit, and plotted against green fluorescence channel. Boxes define $\mathrm{C}-\mathrm{Kit}^{+}$and $\mathrm{c}-\mathrm{Kit}{ }^{+}+$cell populations, and numbers are percentages of gated cells, as mean $\pm \mathrm{S}$.E.M. $(N=25)$. $(h, j)$ The graphs show the number of cells of each population per YS; bars are mean \pm S.E.M,; data were calculated from the total YS cell counts presented in (b). Bars are labeled as in (b). ${ }^{\star *} P<0.01,{ }^{\star * *} P<0.001$ 
described in Figure $3 \mathrm{j}$ is TER119 positive and belongs to the erythroid lineage. The intensity of the CD41/TER119 expression allows the discrimination of primitive and definitive erythroid populations. Most of the erythroid cells found in YS at E9.5 are dim for CD41 and TER119 (Figure $4 \mathrm{e}$ ), therefore corresponding to the primitive wave, a
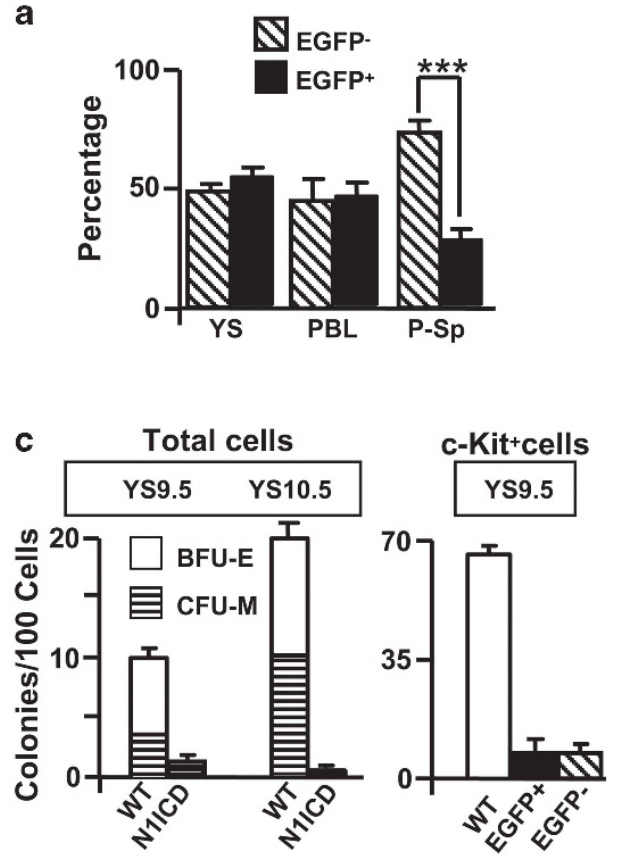

e

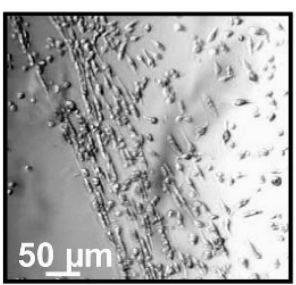

g

TER119-CD41-CD45-MAC1-

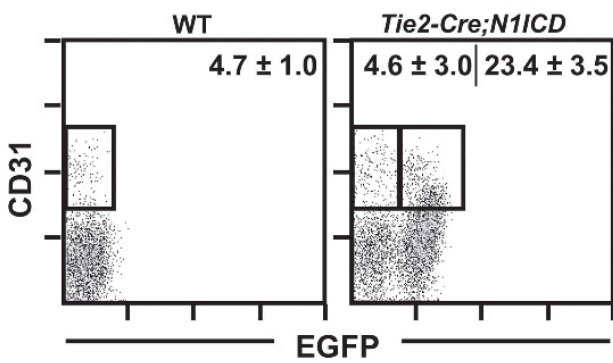

i

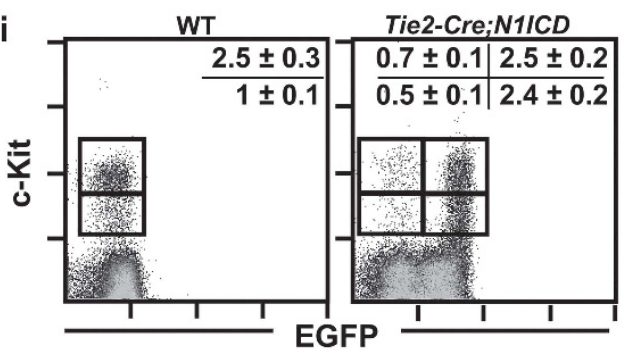

b

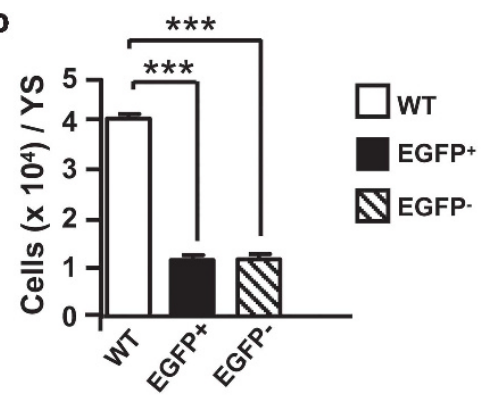

d

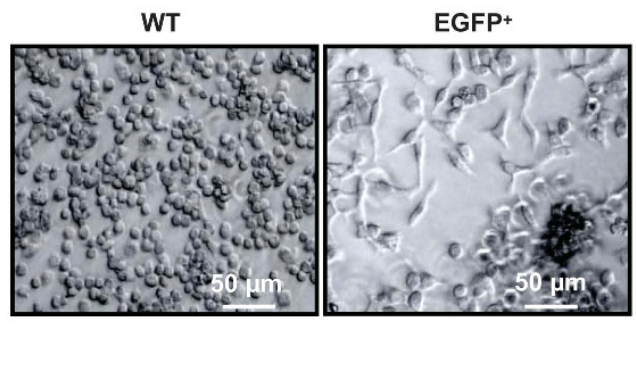

f
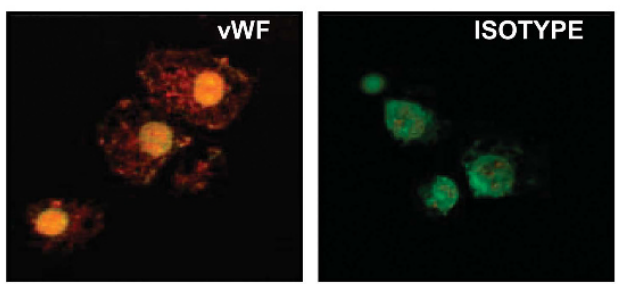

h
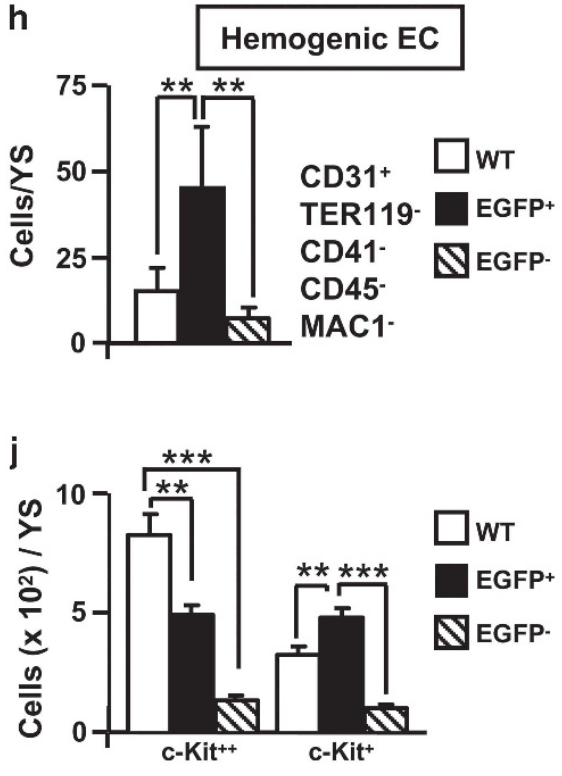

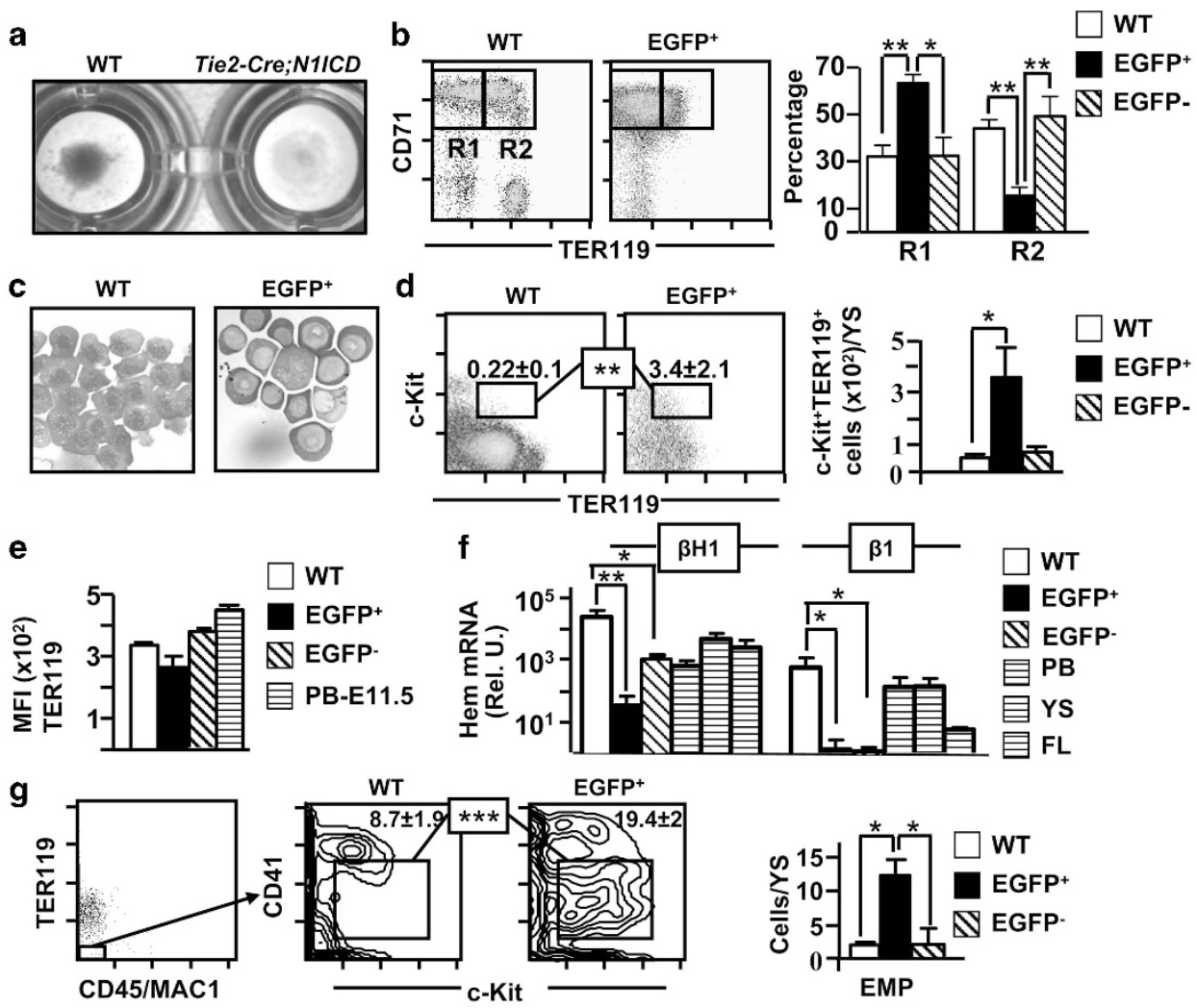

EGFP+ $^{+}$
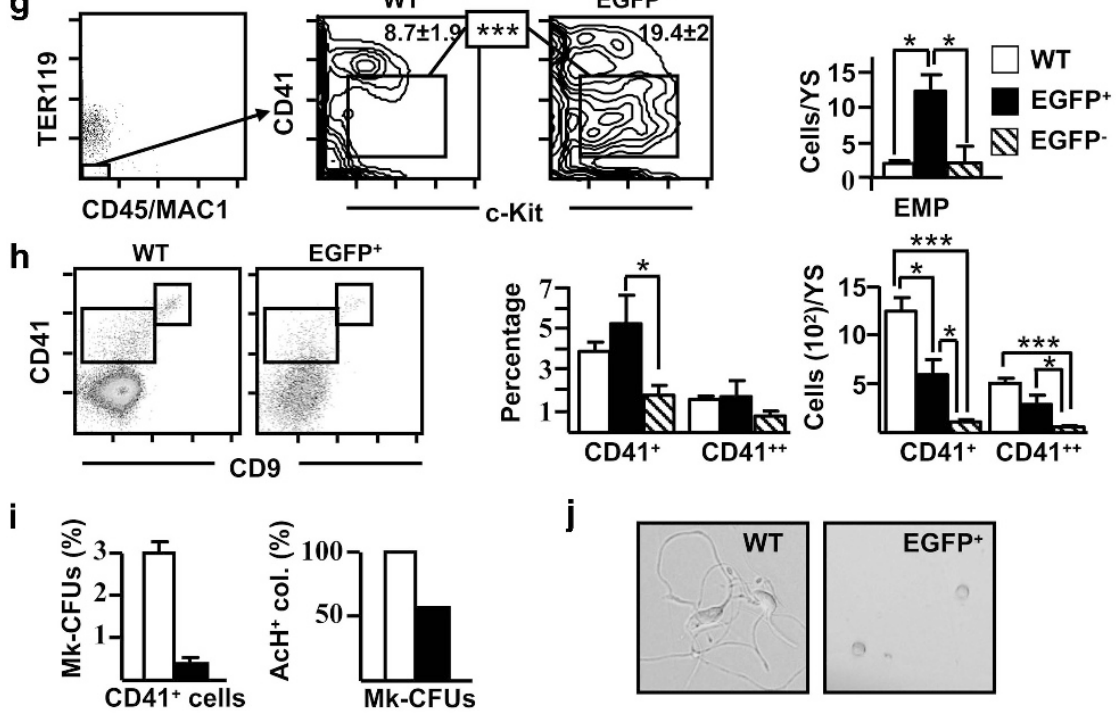

j
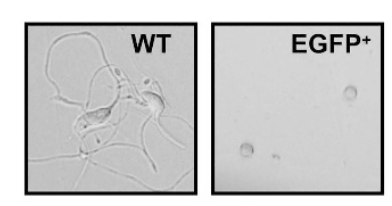

Figure 4 Abnormal erythroid and megakaryocyte differentiation in Tie2-Cre;N1ICDYS. (a) YS cells centrifuged on 96-well plates, revealing the low hemoglobin content of Tie2-Cre;N1ICD YS, indicating defective erythroid differentiation. (b) Representative dot plots of double-stained WT and electronically gated EGFP ${ }^{+}$cells for the erythroid marker TER119 and the transferrin receptor CD71. Boxed areas define CD71 ${ }^{++}$TER $119^{-}$(R1) and CD71 ${ }^{++} /$TER119 ${ }^{+}$(R2) cells. The graph in the right shows the relative percentage of R1 and R2 cell populations (mean \pm S.E.M.; $N=6$ ) in WT and electronically gated EGFP ${ }^{+}$and EGFP- ${ }^{-}$cells. (c) Microphotographs of May GrümwaldGiemsa staining in WT and FACS-purified EGFP ${ }^{+}$Tie2-Cre;N1/CD cells. (d) Representative dot plots of WT and electronically gated EGFP ${ }^{+}$cells for TER119 and c-Kit. Numbers are relative percentages of the TER $119^{+}{ }^{+}$-Kit ${ }^{+}$, cells boxed (mean \pm S.E.M.; $N=6$ ). The right graph shows the quantification of numbers of TER119 ${ }^{+} \mathrm{c}$-Kit ${ }^{+}$ cells/YS calculated as in Figure 3j (mean \pm S.E.M.; $N=6$ ) for WT and electronically gated EGFP ${ }^{+}$and EGFP ${ }^{-}$cells. (e) The graph represents the mean fluorescence intensity (MFI) data obtained from E9.5 WT and electronically gated EGFP ${ }^{+}$and EGFP ${ }^{-}$cells from Tie2-Cre;N1/CD mice stained for TER119, as mean \pm S.E.M. $(N=4)$. Staining of blood cells at E11.5 is shown as a control. (f) Quantifications of embryo-derived primitive $(\beta \mathrm{H} 1)$ and definitive $(\beta 1)$ hemoglobins are shown in the bar graph. WT and FACS-purified EGFP ${ }^{+}$and EGFP- Tie2-Cre;N1/CD cells were submitted to RNA extraction, CDNA was synthesized and RT-qPCR was performed as indicated in Materials and Methods. The Bio-Rad CFX Manager software was used to calculate the $C_{T}$ of each reaction, and the relative amount of specific cDNA in each sample was determined by the $\Delta \mathrm{C}_{\mathrm{T}}$ method. Results are displayed as the relative expression of each transcript over $\mathrm{G} \alpha$ gene expression (mean $\pm \mathrm{S}$.E.M.; $N=4$ ). (g) Representative $\mathrm{FACS}$ analysis of WT and Tie2-Cre;N1/CD mice stained for TER119, CD45/Mac1, CD41 and c-Kit. TER119- CD45/Mac1 ${ }^{-}$cells were gated (left dot plot) and analyzed for CD41 and c-Kit, as indicated in the plots from WT and electronically gated EGFP ${ }^{+}$cells. The boxes identify the c-Kit ${ }^{+} \mathrm{CD}_{4} 1^{+}$erythro-myeloid progenitor (EMP) population; numbers are percentages (mean \pm S.E.M.; $N=4$ ). The right graph shows the EMP quantification/YS calculated as in Figure $3 j$ (mean $\pm S . E . M . ; N=4$ ) for WT and electronically gated $\mathrm{EGFP}^{+}$and EGFP ${ }^{-}$cells. (h) Representative FACS analysis of WT and electronically gated EGFP ${ }^{+}$cells stained for CD9 and CD41. Boxed areas define the doublepositive $\mathrm{CD}^{++}{ }^{+} \mathrm{CD} 41^{++}$megakaryocytic population and the $\mathrm{CD9}{ }^{+} \mathrm{CD} 41^{+}$cell population. The graphs in the right show the relative percentages and absolute numbers of both cell populations in WT and electronically gated EGFP ${ }^{+}$and EGFP ${ }^{-}$Tie2-Cre;N1ICD YS $(N=5)$. (i) Left panel, Representative graph of the relative percentage of megakaryocyte progenitors (MK-CFUs) present in FACS-purified CD41 ${ }^{+}$cells from WT versus EGFP ${ }^{+}$populations $(N=3)$. Right panel, Relative percentages of MK-CFUs expressing acetylthiocholiniodide $(\mathrm{ACH})$ in WT versus EGFP ${ }^{+}$cells $(N=3)$. (j) Microphotographs showing cultures of $\mathrm{CD}{ }^{+}+{ }^{+} \mathrm{CD} 41^{+}+{ }^{+}$FACS-purified cells from E9.5 WT and EGFP ${ }^{+}$Tie2-Cre;N1ICD YS cells at $48 \mathrm{~h}$ of culture. ${ }^{*} P<0.05,{ }^{* *} P<0.01,{ }^{* * *} P<0.001$ 
as has been described. ${ }^{15}$ However, EGFP ${ }^{+}$Tie2-Cre;N1ICD cells displayed lower levels of TER119 than WT cells. Accordingly, the expression of both the fetal $\beta \mathrm{H} 1$ and the adult-type $\beta 1$ hemoglobin was highly diminished in EGFP ${ }^{+}$ Tie2-Cre;N1ICD cells compared with WT cells (Figure 4f). Finally, the quantification of the more immature $\mathrm{c}-\mathrm{Kit}^{+}$ CD41 ${ }^{+}{\text {CD } 45{ }^{-} \text {Mac1 }^{-} \text {TER119 }}^{-}$common erythro-myeloid progenitors $^{41}$ by 6 -color cytometry showed that they were significantly increased in EGFP ${ }^{+}$cells compared with WT cells (Figure 4g). In summary, constitutive N1ICD expression on $\mathrm{Tie} 2^{+}$cells blocks in the YS the maturation of erythroid precursors both of the primitive erythropoiesis wave and of the definitive transient erythro-myelopoiesis, resulting in an accumulation of erythro-myeloid progenitors and of immature proerythroblasts. The EGFP ${ }^{-}$erythroid cells present in Tie2Cre;N1ICD YS had phenotypes similar to WT cells, although their total numbers were reduced (Supplementary Figure $4 a$ and $\mathrm{b})$. The expression levels of adult-type $\beta 1$ hemoglobin were strongly reduced, whereas the levels of fetal $\beta \mathrm{H}$ in EGFP $^{-}$cells were lower compared with WT cells, this may be related with the smaller size of the EGFP ${ }^{-}$erythroid cell compartment (Figure 4f).

Besides erythroid cells, the first hematopoietic-lineage cells generated in the $\mathrm{YS}$ belong to the megakaryocyte (MK) lineage. ${ }^{15,42}$ WT and Tie2-Cre;N1ICD YS did not differ in relative numbers of $\mathrm{CD} 41^{++} \mathrm{CD} 9^{++}$cells, which correspond to embryonic MKs (Figure 4h). ${ }^{42}$ Nevertheless, fewer total $\mathrm{EGFP}^{+}$and EGFP ${ }^{-} \mathrm{CD} 41^{++} \mathrm{CD}^{++}$MKs were present in Tie2-Cre;N1ICD YS compared with WT YS. Purified EGFP ${ }^{+}$ $\mathrm{CD} 41^{++} \mathrm{CD}^{++}$cells cultured in semisolid MK-specific medium produced fewer MK-CFUs than their WT counterparts, and $50 \%$ of them contained immature acetylthiocholiniodide ${ }^{-}$ cells (Figure 4i). After $24 \mathrm{~h}$ in liquid culture, purified CD41 ${ }^{++}$ $\mathrm{CD}^{++}$cells from WT and Tie2-Cre;N1/CD embryos began to differentiate to MKs, exhibiting cytoplasmic prolongations. However, only WT MKs developed into pro-platelets at $48 \mathrm{~h}$, whereas $\mathrm{EGFP}^{+}$MKs disappeared from the cultures (Figure 4j). Thus, regardless of the enrichment in erythromyeloid progenitors and the relatively normal numbers of $\mathrm{CD} 4{ }^{++} \mathrm{CD}^{++} \mathrm{MK}$ progenitors in the transgenic YS, their differentiation in vitro is defective and MK progenitors die before reaching their final maturation stage.

Phenotypic characterization of myeloid immature progenitor cell populations in WT and Tie2-Cre;N1ICD YS at E9.5. To characterize the myeloid differentiation potential of WT and Tie2-Cre;N1ICD YS cells at E9.5, we analyzed the co-expression of c-Kit and CD45, which allowed us to trace the appearance of myeloid CD45 ${ }^{+}$cells in the $\mathrm{YS} .^{39}$ Similar numbers of $\mathrm{EGFP}^{+} \mathrm{CD} 45^{+}$cells and WT CD45 ${ }^{+}$ cells were detected (Figure 5a). However, the EGFP ${ }^{+}$ $\mathrm{CD}^{+} 5^{+}$population from Tie2-Cre;N1ICD YS contained a smaller immature $\mathrm{c}-\mathrm{Kit}^{++} \mathrm{CD}^{+} 5^{+}$(R1) fraction (Figure $5 \mathrm{~b}$ ). In contrast, the numbers of $\mathrm{c}-\mathrm{Kit}^{+}{ }^{+}$cells with higher CD45 levels (R2, c-Kit ${ }^{++} \mathrm{CD}_{4} 5^{++}$) remained similar in WT and $\mathrm{EGFP}^{+} \mathrm{YS}$. The more mature intermediate $\mathrm{C}-\mathrm{Kit}^{+} \mathrm{CD} 45^{++}$ (R3) cells, showing loss of c-Kit, accumulated in EGFP ${ }^{+} \mathrm{YS}$, whereas the numbers of more mature $\mathrm{c}-\mathrm{Kit}^{-} \mathrm{CD} 45^{++}$cells (R4) were similar in the two genotypes (Figure 5b). Expression analysis of the macrophage marker $\mathrm{F} 4 / 80^{43}$ detected similar numbers of $\mathrm{F} 4 / 80^{+}$cells in WT samples and EGFP ${ }^{+}$cells from Tie2-Cre;N1ICD YS (Figure 5c). However, the WT $\mathrm{F} 4 / 80^{+}$YS population contained a subpopulation of $\mathrm{CD}_{4} 5^{++}$cells expressing the myeloid marker Mac1 Mac1 $^{++}$ $\mathrm{CD}_{4}{ }^{++}$) that was absent in $\mathrm{F} 4 / 80^{+} \mathrm{EGFP}^{+}$cells (Figure 5c); moreover, $\mathrm{Mac1}^{++}$cells were found only in WT YS (Figure 5d), whereas the EGFP ${ }^{+}$YS population contained elevated numbers of immature $\mathrm{Mac1}^{+}$cells. $\mathrm{Mac1}^{++}$cells in WT YS are mature myeloid cells, since they were negative for C-Kit, and expressed Gr1 and high levels of CD45 (Figures $5 d$ and e). Thus, N1ICD expression on $\mathrm{Tie} 2{ }^{+}$cells does not affect the initiation of myelopoiesis, but impairs developmental progression to the final maturation stages, and consequently causes accumulation of intermediate c-Kit ${ }^{+}$CD $45^{++}$stages. The myeloid differentiation potential of EGFP $^{-}$Tie2-Cre;N1ICD YS cells was also altered, as reflected by the smaller numbers of EGFP ${ }^{-} \mathrm{CD}_{4}{ }^{+}$cells and of EGFP ${ }^{-} \mathrm{F} 4 / 80^{+}$cells and by the lower percentages of immature myeloid cellular stages (R1 and R2; Figure 5b). Interestingly, the fact that fractions of more mature $\mathrm{Mac1}{ }^{++}$ $\mathrm{Gr}^{+}$cells were detected at similar level in EGFPTie2-Cre;N1ICD YS cells than in WT preparations, suggested that EGFP ${ }^{-}$cells were able to reach final myeloid development stages (Supplementary Figure 4d and e).

Notch1 activation on Tie2-derived YS cells dysregulates the transcriptional program that initiates hematopoietic differentiation. Analysis of FACS-purified EGFP ${ }^{+}$and WT YS cells by quantitative PCR ( $\mathrm{PPCR}$ ) revealed an association of N1ICD expression with substantial changes in expression of hematopoietic and vasculo/endothelial genes. EGFP ${ }^{+}$ cells expressed higher levels of Runx1 (Figure 5f). The transcription factors SCL, Fli1 and Gata family members are implicated in the earliest stages of hematopoiesis/vasculogenesis in the mouse embryo, and have been proposed to form a gene regulatory network that operates during specification of vasculo-hematopoiesis. ${ }^{44}$ EGFP $^{+}$cells expressed lower levels of Scl, Gata3 and Fli1 transcripts, whereas Gata1 and Gata2 were upregulated (Figure 5f). The transcript levels of $P u 1$ and $c$-fms, which have a critical role in differentiation to myeloid lineages, ${ }^{45}$ were increased in $\mathrm{EGFP}^{+}$cells. In contrast, transcripts encoding myeloperoxidase and lysozyme, involved in differentiation of more mature myeloid stages, were strongly downregulated, in agreement with the incomplete myeloid development observed in $\mathrm{EGFP}^{+}$Tie2-Cre;N1/CD cells. ${ }^{43}$ Despite the marked alteration in erythropoiesis observed in Tie2-Cre;N1ICD embryos, the transcription of Klf1 and KIf2, important genes in this process ${ }^{46}$ did not show variations. Constitutive Notch1 activation in Tie2-derived cells thus dysregulates the transcriptional program that initiates the first differentiation steps in early hematopoietic progenitor cells and some lineage-restricted precursors.

N1ICD expression in Tie2 ${ }^{+}$cells induces high proliferation, cell-cycle activation and reduced apoptosis. Notch1 activation has been shown to enhance self-renewal, increases the stem-cell pool, alters differentiation processes and promotes cell-cycle progression. ${ }^{24}$ The erythroid/myeloid differentiation arrest that we found at E9.5 in EGFP ${ }^{+}$cells 
derived from $\mathrm{Tie}{ }^{+} \mathrm{N} 1 \mathrm{ICD}$ expressing progenitors was accompanied by an increase in the number of erythroid/ myeloid $\mathrm{C}^{-\mathrm{Kit}^{+}}$immature cell progenitors. Also, FACSpurified EGFP ${ }^{+}$Tie2-Cre;N1ICD cells cultured on OP9 grew at a 10-fold higher rate than WT or EGFP ${ }^{-}$Tie2-Cre;N1ICD cells (Figure 6a). Analysis of the cell-cycle status of purified EGFP $^{+}$cells by propidium iodide (PI) staining revealed a higher number of cells in the $S / G_{2} M$ phases compared with
WT cells (Figure 6b). This was confirmed also for WT and Tie2-Cre;N1/CD cells by Hoechst staining of the nuclei of live cells, which allows ex vivo identification cell-cycle status, revealing a higher proportion of $\mathrm{EGFP}^{+}$cells in $S$ phase (Figure $6 \mathrm{c}$ ) than WT or EGFP ${ }^{-}$Tie2-Cre;N1ICD cells. In contrast, in vivo incorporation of the nucleotide analog EdU did not differ between EGFP ${ }^{+}$and WT cells (Figure $6 \mathrm{~d}$ ). To exclude the influence of gestational age differences between
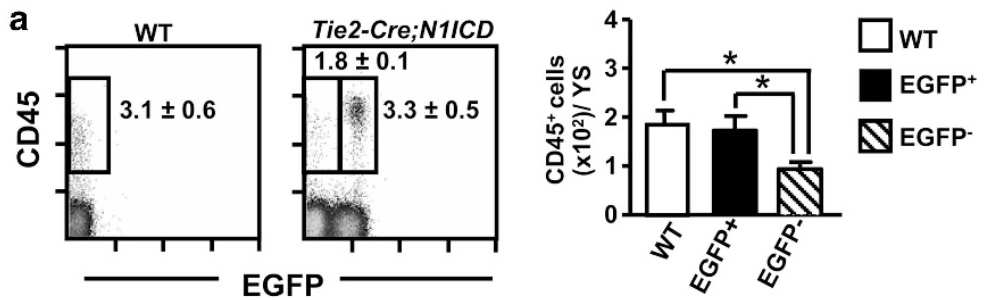

b
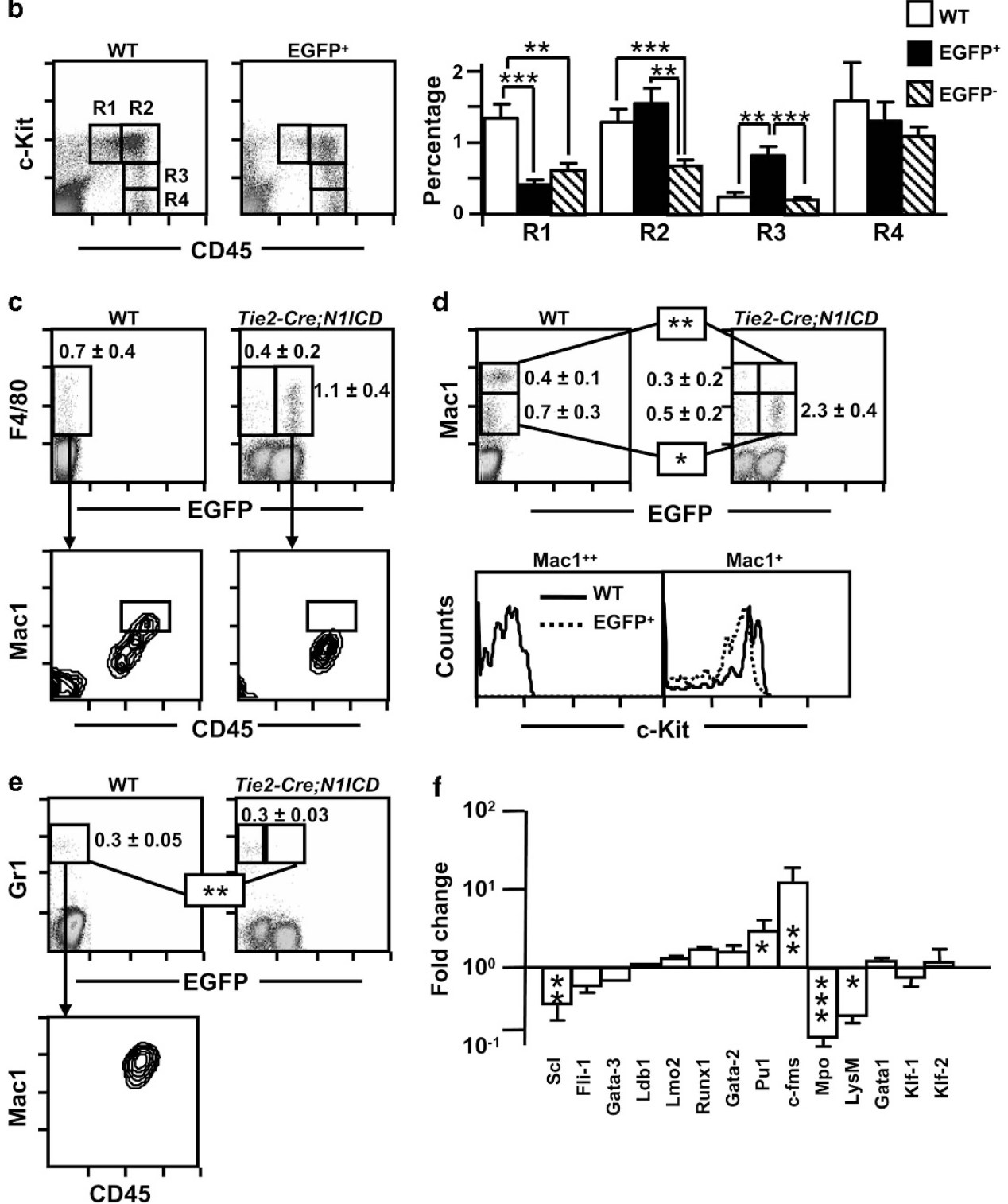
mice, we compared EdU incorporation between EGFP ${ }^{+}$and EGFP $^{-}$cells in the same Tie2-Cre;N1/CD embryos, and found that cells expressing the transgene incorporated more EdU than EGFP ${ }^{-}$cells. Apoptotic cells, defined as those in the $\leq \mathrm{G}_{0}$ region and by the expression of Annexin- $\mathrm{V}$, were less abundant in total EGFP ${ }^{+}$cells than in total WT cells or in EGFP ${ }^{-}$Tie2-Cre;N1/CD cells (Figure 6e). These data thus indicate that N1ICD expression in $\mathrm{Tie} 2^{+}$cells prevents apoptosis and induces a highly proliferative state in most YS hematopoietic progenitors, with an accumulation of cells in the $S$ phase, which likely interferes with their differentiation.

Analysis of Notch pathway genes by qPCR revealed that Notch1, Hey2, Hes1, Jag1, Jag2, DIl1 and DIl4 were upregulated in EGFP ${ }^{+}$cells relative to WT cells, consistent with an increase in Notch signaling activity (Figure 6f). Interestingly, EGFP ${ }^{-}$Tie2-Cre;N1ICD cells showed equivalent levels to those from WT cells, except for Jag2, which was upregulated. Among the regulators of the cell-cycle machinery, both Cyclin-D1 and $c-m y b$ were induced in EGFP ${ }^{+}$cells relative to WT cells, whereas the cell-cycle inhibitor p27 was downregulated. No significant changes were observed for c-myc, Id/Atp8b2 or p21 (Supplementary Figure 4f).

\section{Discussion}

The aim of this study was to determine whether Notch function in embryonic hematopoiesis is restricted to the definitive hematopoietic compartment, as has been proposed based on loss-of-function approaches. ${ }^{47}$ The Tie2 promoter is active in endothelial and hematopoietic lineages from E7.5 onwards, ${ }^{48}$ and almost all primitive hematopoietic cells can be traced to $\mathrm{Tie}^{+}$cells. N1ICD and Tie2 have a similar expression pattern in YS blood islands. ${ }^{29,30}$ As a strategy to induce Notch1 in embryonic hematopoietic cells we generated double transgenic embryos constitutively expressing N1ICD in $\mathrm{Tie} 2^{+}$hematovascular progenitor cells. Our results show that constitutive N1ICD expression in Tie $2^{+}$hematopoietic progenitors stimulates cellular proliferation at the expense of differentiation, disturbing the delicate balance between both processes and leading to a block of hematopoietic differentiation while promoting the accumulation of cells of intermediate phenotypes (Figure 7).
It is well established that Tie2 is required for primitive and definitive hematopoiesis. ${ }^{49}$ We have observed that Tie2Cre; $N 1 / C D$ mice show around $50 \%$ of EGFP ${ }^{-}$cells, indicating that Tie2-Cre-driven recombination is, as previously reported, ${ }^{50}$ incomplete. EGFP ${ }^{-}$cells express low fractions of Tie2, similarly to WT cells, and although in EGFP ${ }^{+}$cells the expression of Notch1 and related genes is highly increased, in EGFP $^{-}$cells it is close to that from WT, except for Jag2. Therefore, although we cannot completely exclude that some $\mathrm{EGFP}^{-}$cells derive from rare Tie2-independent hematopoietic cells, they are very likely the result of incomplete Cre recombination. Our data on EGFP ${ }^{-}$cells provide evidence of a non-cell autonomous effect of Notch1, probably acting through the Jag ligand. In fact, EGFP ${ }^{-}$cells have reduced numbers and seem to have altered $\mathrm{c}-\mathrm{Kit}^{++}$progenitor cells and differentiation in vitro. It seems that, in vivo, once the $\mathrm{c}-\mathrm{Kit}^{+}$cells overcome their differentiation block, they are able to proceed to normal primitive erythro-myelopoiesis (erythroid/MK differentiation, and $\mathrm{CD}_{4} 5^{+} \mathrm{Mac} 1^{++} \mathrm{Gr} 1^{+}$ myelopoiesis), although always maintaining reduced numbers of lineage-specific cells.

Previous reports showed an essential role of Notch in the onset of definitive hematopoiesis in the mouse P-Sp/AGM region, but with little or no effect on the primitive hematopoietic wave. ${ }^{26,35-37}$ In contrast, our gain-of-function approach has not only allowed us to determine that adult-type P-Sp/AGM hematopoiesis is impaired in Tie2-Cre; N1/CD embryos, but also revealed severe alterations in $\mathrm{YS}$-derived primitive and transient definitive hematopoiesis. At E9.5, the YS of transgenic embryos contains above-normal numbers of c-Kit-low expressing cells, and low numbers of immature ${\mathrm{c}-\mathrm{Kit}^{+}}^{+}$cells that have a weak hematopoietic potential in vitro. Constitutive Notch1 activation in Tie $2^{+}$cells moreover leads to the maintenance in the $\mathrm{YS}$ of $\mathrm{CD} 31^{+} \mathrm{CD} 41^{-}$ CD45 ${ }^{-}$Mac1 $^{-}$TER119 ${ }^{-}$hemogenic endothelial cells and of $\mathrm{C}-\mathrm{Kit}^{++} \mathrm{Tie}^{+}$progenitors with in vitro endothelial potential, bearing a c-Kit ${ }^{++} /^{+} \mathrm{Tie} 2{ }^{+} \mathrm{CD} 31^{+} /^{-} \mathrm{KDR}^{-}$phenotype.

The in vivo mapping of Notch pathway activity in adult lymphoid tissues and in YS from E10.5 has being recently reported. ${ }^{51}$ No Hes1 expression on embryo HSC was found, despite Notch $1 / 2$ expression, suggesting that Notch signaling was not activated. MKs and erythrocytes expressed active

Figure 5 N1ICD inhibits the differentiation of YS myeloid progenitors. Total WT and Tie2-Cre;N1/CD YS cells were stained with the indicated antibodies and analyzed by flow cytometry. (a) $\mathrm{CD} 45^{+}$cell numbers are elevated in YS from Tie2-Cre; N1/CD mice at E9.5. Dot plots show a representative detection of myeloid CD45 ${ }^{+}$cells (boxed areas) in WT and Tie2-Cre;N1ICD preparations. Inside the plots cell percentages are shown as mean \pm S.E.M., $N=15$. The bar chart shows the number of CD45 ${ }^{+}$cells per YS, calculated for each sample using the corresponding data presented in Figure $3 \mathrm{~b}$. (b) Dot plots of WT and electronically gated EGFP ${ }^{+}$YS cells stained for the hematopoietic markers CD45 and c-Kit. Gates define the following subpopulations: c-Kit ${ }^{++} \mathrm{CD}^{+} 5^{+}$(R1), c-Kit ${ }^{++} \mathrm{CD}^{+} 5^{++}$(R2), $\mathrm{c}-\mathrm{Kit}^{+} \mathrm{CD} 45^{++}$(R3) and c-Kit $\mathrm{CD}^{+} 5^{+}$(R4). The bar chart shows relative percentages of electronically gated WT and EGFP ${ }^{+}$and EGFP ${ }^{-}$Tie2-Cre;N1/CD YS cells as mean \pm S.E.M., N $=10$. (c) Upper panel: dot plots show a representative detection of the macrophage marker $F 4 / 80$, boxed areas with relative percentages as mean ( \pm S.E.M., $N=4$ ), in total WT and Tie2-Cre;N1/CD YS cells, detected in the green fluorescence channel to identify EGFP ${ }^{+}$cells. Lower panel: dot plots show Mac1 and CD45 expression in electronically gated WT F4/80 ${ }^{+}$cells and EGFP ${ }^{+} \mathrm{F} 4 / 80^{+}$cells. The boxed areas highlight a Mac1 ${ }^{++}{ }^{+} \mathrm{CD} 45^{++}$subpopulation not found among EGFP ${ }^{+} \mathrm{F} 4 / 80^{+}$cells. (d) Mac1 antibody staining in total WT and Tie2-Cre;N1/CD YS cells, displayed as in (c). The boxes mark the Mac1 ${ }^{+}$and Mac1 ${ }^{++}$subpopulations, with relative numbers shown to the right as mean \pm S.E.M., $N=4$; Mac1 $^{++}$cells are absent in EGFP ${ }^{+}$transgenic mice. The lower histograms show c-Kit-stained cells in the electronically gated Mac1 ${ }^{+}$and $\mathrm{Mac1}^{++}$subpopulations from WT or EGFP ${ }^{+}$YS. Mac1 ${ }^{+}$cells in WT (solid line) and EGFP ${ }^{+}$(open line) YS cells (left histogram) co-express c-Kit. WT Mac1 ${ }^{++}$cells do not express c-Kit, indicating their more mature phenotype (right histogram). (e) Dot plots of Gr1 staining (boxed areas) showing the absence of Gr1 ${ }^{+}$cells in the EGFP ${ }^{+}$ Tie2-Cre; N1ICD YS cells. The percentage of $\mathrm{Gr}^{+}$cells is indicated as mean \pm S.E.M., $N=3$. The contour plot (bottom) shows co-expression of Mac1 and CD45 in electronically gated WT Gr1 ${ }^{+}$cells. (f) Quantitative PCR analysis of FACS-purified WT and EGFP ${ }^{+}$cells for the expression of selected genes implicated in early hematopoiesis (SCL, Fli1, GATA3, Ldb1, Lmo2 and Runx1), myeloid development (GATA2, PU.1, c-fms, Mpo and LysM) and erythroid development (GATA1, KLF1 and KLF2). Data are shown as the fold change in mRNA expression level ( $\triangle \Delta C$ t method) between EGFP ${ }^{+}$cells from Tie2-Cre;N1ICD YS relative to WT YS cells. Bars are mean \pm S.E.M., $N=3 .{ }^{*} P<0.05,{ }^{* *} P<0.01,{ }^{* \star *} P<0.001$ 
a

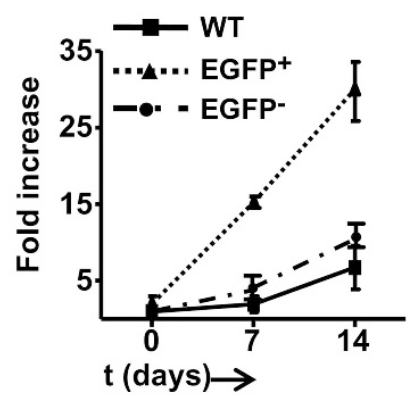

b

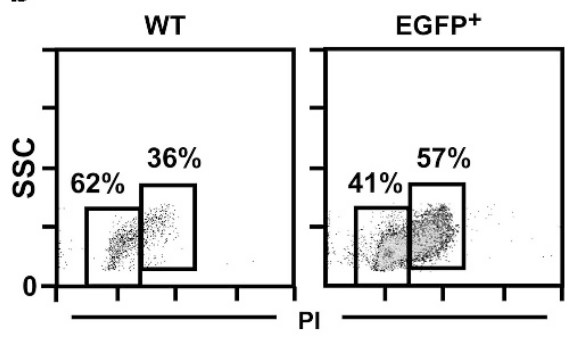

C
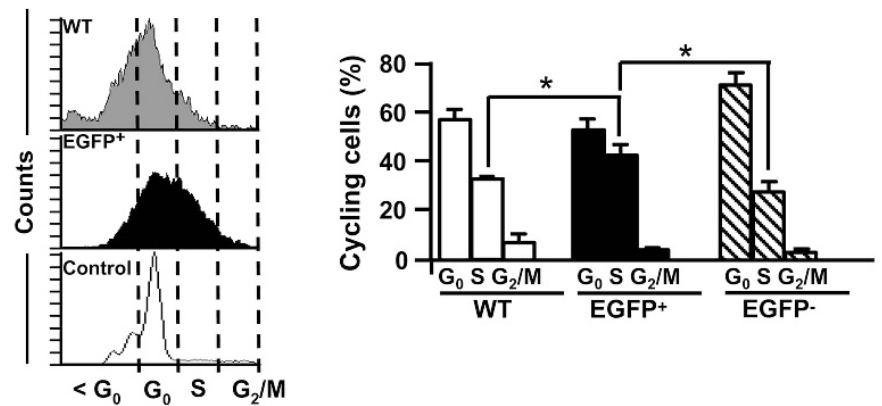

d

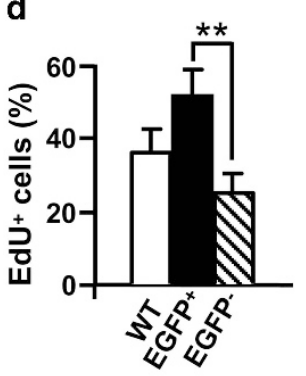

e

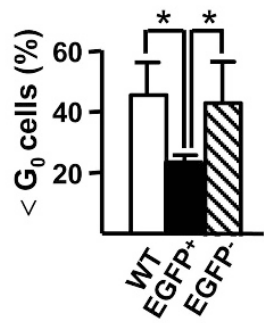

Annexin V
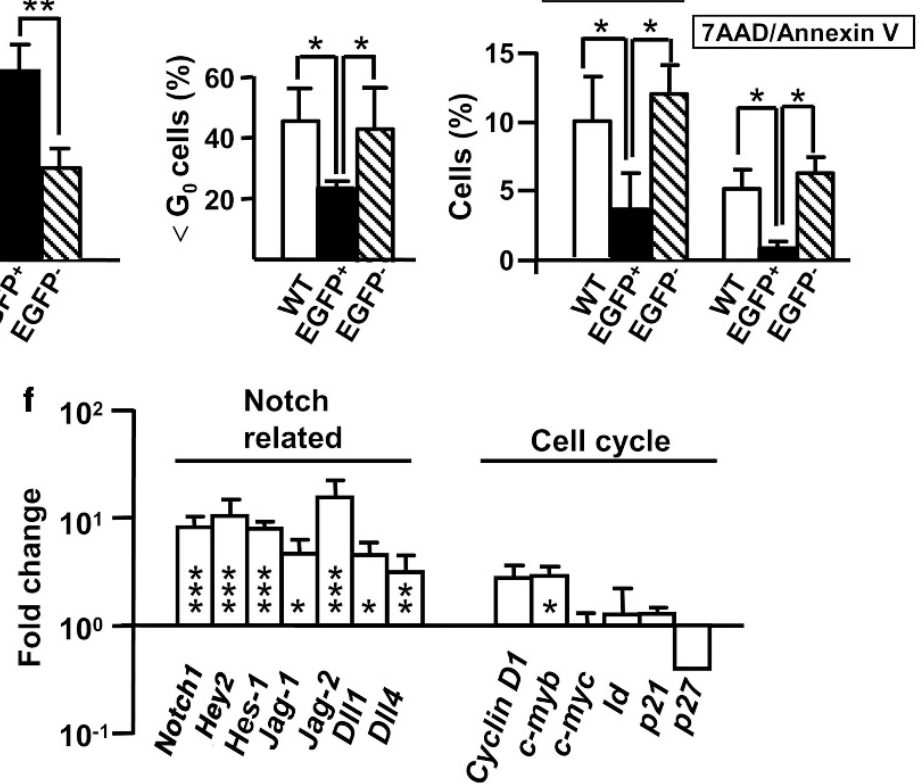

Figure 6 Proliferation, cell cycle and apoptosis in Tie2-Cre;N1/CD YS. (a) Equal numbers ( $2 \times 10^{3}$ cells/well) of WT or Tie2-Cre;N1ICD YS cells were cultured on OP9 stromal cells. Cells were recovered after 7 days, counted, replated at $2 \times 10^{3}$ cells/well and cultured for further 7 days. The percentage of EGFP ${ }^{+}$cells was detected by flow cytometry, and absolute numbers of each cell type were calculated from the total number of recovered cells. Fold increase in cell number was calculated at 7 and 14 days. Mean \pm S.E.M., $N=3$. (b) Representative dot plots of FACS-purified WT and EGFP ${ }^{+}$Tie2-Cre;N1/CD cells, showing cell size (SSC) and propidium iodide staining for cell-cycle analysis. Boxed areas define $G_{0} / G_{1}$ phase (left box) and the $S$ plus $G_{2} M$ phase populations (right box), and numbers are the percentages in this experiment ( $N=2$ ). (c) DNA content quantified by cytometric analysis of electronically gated EGFP ${ }^{+}$Tie2-Cre;N1ICD and WT cells stained with Hoechst 33342. Representative histograms are shown for WT, EGFP ${ }^{+}$and EGFP ${ }^{-}$YS cell populations. Vertical dotted lines indicate the cell-cycle phases defined by the control cells used (adult thymus). The bar chart shows cells in $G_{0} / G_{1}, S$ and $G_{2} M$ as a percentage of total cells found in cycle $\left(>G_{0}\right)(N=4)$. (d) Relative percentage of EGFP ${ }^{+}$, EGFP ${ }^{-}$and WT cells that incorporated the nucleotide-analog EdU $1 \mathrm{~h}$ after intraperitoneal injection into the pregnant females ( $N=6$ for cells purified from YS of Tie2-Cre;N1/CD mice and $N=11$ for WT YS cells). (e) Left panel, Percentages of apoptotic cells ( $<G_{0}$ cell population) detected in the DNA content cytometry analysis shown in (c) in WT and EGFP ${ }^{+}$YS cells. Right panel, Percentages of apoptotic WT and EGFP ${ }^{+}$and EGFP ${ }^{-}$YS cells defined by cytometric analysis of staining with Annexin V and 7-aminoactinomycin D (7AAD) (N=4). (f) Fold change ( $\triangle \Delta \mathrm{Ct}$ method) in mRNA expression levels of Notch pathway and cell-cycle regulatory genes in FACS-purified EGFP ${ }^{+}$Tie2-Cre;N1/CD cells relative to WT cells. The data were obtained as in Figure $5 f$ for each sample. ${ }^{*} P<0.05$, ${ }^{* \star} P<0.01$, ${ }^{\star \star *} P<0.001$

Notch2, whereas much higher Notch1 levels were found in endothelial $\mathrm{CD} 31^{+}$precursors. The in vitro transduction of constitutive active truncated Notch2 in $\mathrm{CD} 34^{+}$human cells showed increased numbers of basophilic immature erythroblast that did not reach mature stages. ${ }^{52} \mathrm{~A}$ report using an N1ICD overexpression system in $\mathrm{Tie}^{+}{ }^{+}$cells similar to ours 


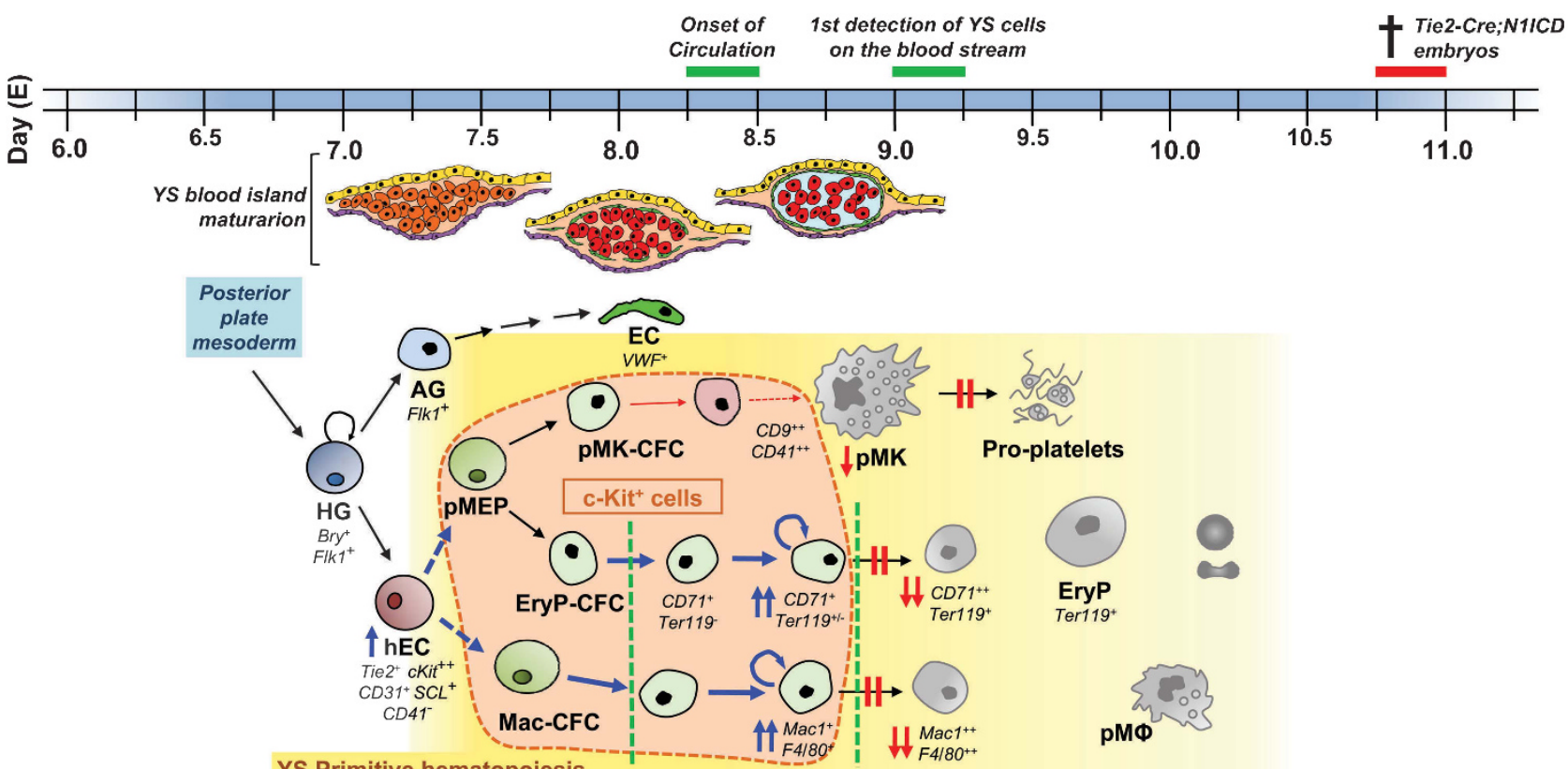

YS Primitive hematopoiesis

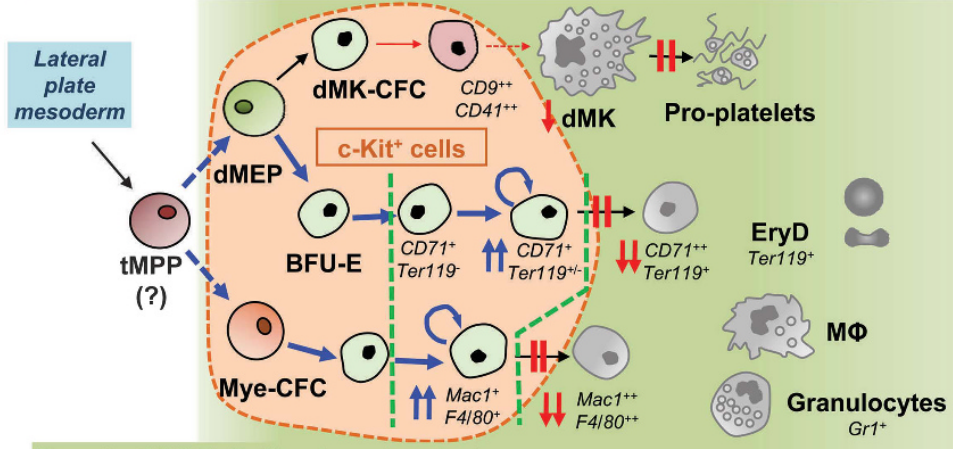

Transient YS Definitive hematopoiesis

Figure 7 Yolk sac hematopoiesis alterations in Tie2-Cre;N1/CD embryos. In the mouse at around embryonic day (E) 7.0-7.25 early hemangioblasts (HG) emerged from the posterior streak mesoderm and give rise to hemogenic endothelial cells ( $\mathrm{hEC}$ ), and angioblasts (AG) progenitor cells on the extraembryonic YS blood island. The AG will produce part of the YS vascular endothelial cells (EC). On the other hand, YS hematopoiesis occurs in two waves, the primitive (from $\sim E 7.25$, yellow box) and definitive (green box) waves that overlap, temporally and spatially, and produce relatively short-lived cells. $\mathrm{hEC}$ give rise, first, to bipotential primitive megakaryocyte/erythroid progenitor (pMEP) that will in turn generate specific primitive progenitors for both cell lineages (pMK-CFC and EryP-CFC); and second, to primitive macrophage precursor cells (Mac-CFC). These progenitors will generate the primitive hematopoietic cell lineages: primitive erythrocytes (EryP), primitive megakaryocytes (pMK) - able to give rise to proplatelets - and primitive macrophages (pMФ). This short-lived primitive wave is rapidly followed by the second hematopoietic wave that comprises YS definitive erythroid, megakaryocyte and several myeloid lineages. The YS definitive populations are believed to arise from transient multipotent progenitors (tMPPs) - but not from hematopoietic stem cells, which emerge in the mouse YS at around E9.0 - that are detected from E8.25 and generate different transient cell populations of restricted erythro-myeloid progenitors (EMPs). EMPs comprise definitive-type bipotential megakaryocyte/erythroid progenitor (dMEP) that will give rise to transient definitive megakaryocyte and erythrocyte progenitors (dMK-CFC and BFU-E) and definitive myeloid progenitors (Mye-CFC), from which definitive-type erythrocytes (EryD), megakaryocytes (dMK) - with the capacity to originate proplatelets - and several myeloid lineages: macrophages $(M \Phi)$, granulocytes and mast cells will be produced. The expression of N1ICD in early $\mathrm{Tie}^{+}$progenitors produces severe alterations in both $\mathrm{YS}^{-}$primitive and definitive hematopoiesis characterized by high frequency of c-Kit-expressing multipotential and lineage-restricted progenitors (encircled by the orange bubbles) at E9.5, whereas it blocks the generation of the last maturation stages of erythrocytes, megakaryocytes and myeloid lineages. First, no $\mathrm{CD} 71^{++} \mathrm{TER} 119^{+}$and $\mathrm{Mac1}^{++} \mathrm{F} 4 / 80^{++}$cells are produced. In contrast, lineage-restricted progenitors accumulate in the initial or intermediate stages of their differentiation (CD71 ${ }^{++}$TER $119^{-}$proerythroblasts and Mac1 $1^{+} \mathrm{F} 4 / 80^{+}$myeloid progenitors). Accordingly, diminished numbers of $\mathrm{CD} 9^{+}$ ${ }^{+} \mathrm{CD} 41^{++}$megakaryoblasts are found among EGFP ${ }^{+}$YS cells and the final steps of differentiation in vitro are selectively blocked. On the other hand, ectopic N1ICD expression in these intermediate progenitors (delimited by green open lines) induces misbalanced proliferation and apoptosis, preventing their normal differentiation. Thick blue arrows denote an increase in cell frequency; weak red arrows indicate a restriction on cell frequency

also found, as we do, a block in erythroid cell development at the level of proerythroblasts, but quantification of the Ly6 $\mathrm{C}^{+}$ myeloid cells led the authors to conclude that myeloid differentiation was preserved..$^{53}$ In our system, an extensive study of lineage markers and functional differentiation assays showed that N1ICD overexpression in Tie2 ${ }^{+}$cells produces a defect in the maturation process of the erythroid, megakaryocytic and myeloid lineages that prevents them from reaching the last maturation stages, resulting in an accumulation of erythroid and myeloid cells at different stages of differentiation, including erythro-myeloid progenitors. A similar defect in the in vitro maturation of MKs has recently been described following activation of DII4-Notch signaling in human cord blood $\mathrm{CD} 34^{+}$cells. ${ }^{54}$ In agreement with our 
results, N1ICD activation has been demonstrated to inhibit myeloid differentiation and to induce early stages of T-cell development from HSCs. ${ }^{55}$

Our data additionally show that expression of N1ICD in $\mathrm{Tie}^{+}$cells prevents apoptosis and disrupts the proliferation and differentiation balance of embryonic hematopoietic cell lineages. Since the equilibrium between cellular proliferation and differentiation is strictly regulated, ${ }^{56}$ any alteration will affect lineage development. Our results suggest that Notch1 may normally function to fine-tune this process. Thus in transgenic YS, lineage-restricted progenitors (erythroblasts, megakaryoblasts and myeloblasts) would be unable to differentiate because of a continued proliferative stimulus elicited by constitutive N1ICD activity. This results in low numbers of HSCs and the accumulation of different intermediate progenitors, while impeding terminal differentiation of endothelial, erythroid, MK and myeloid cells. This phenotype would be compatible with a stem-cell exhaustion process that would occur early during primitive hematopoiesis. ${ }^{56}$ In conclusion, our results show an unrevealed role of Notch signaling in YS-derived hematopoiesis by interfering in the proliferation and differentiation processes of lineagerestricted intermediate progenitors, producing a block of terminal differentiation.

\section{Materials and Methods \\ Transgenic mice, embryo dissection and cell suspensions. Embryos overexpressing N1ICD-EGFP in Tie2-derived cells (Tie2-Cre;N1/CD) and their WT littermates were obtained by crossing Rosa26-N1/CD-EGFP ${ }^{32}$ (for simplicity N1/CD) with Tie2-Cre transgenic mice. ${ }^{31}$ Mice were maintained in the animal facility of the Centro Nacional de Investigaciones Cardiovasculares under pathogen-free conditions. All animal procedures conformed to EU Directive 2010/ 63EU and Recommendation 2007/526/EC regarding the protection of animals used for experimental and other scientific purposes, enforced in Spanish law under Real Decreto 1201/2005. Embryos were dissected and exsanguinated, and the embryonic blood and tissues (YS and the caudal portion of the embryo containing the P-Sp/AGM region) were recovered as described. ${ }^{57}$ Cell suspensions were prepared from pooled embryonic tissues of the same gestational age by mechanical dissociation and cells were counted in a Neubauer chamber.}

Flow cytometry analysis and cell sorting. The monoclonal antibodies (mAbs) used are detailed in Table 1. Cell suspensions were incubated with blocking solution (Fc block; BD Biosciences Inc., San José, CA, USA) for 10 min at $4^{\circ} \mathrm{C}$. After washing, the cells were incubated with biotinylated, PE-, allophycocyanin-, PerCP-cyanin5.5 or PE-cyanin7-conjugated mAbs. Biotinylated mAbs were revealed by $\mathrm{PE}$, allophycocyanin or $\mathrm{PE}$-cyanin7-streptavidin. Cell debris and dead cells were discarded by 4',6-diamidino-2-phenylindole (DAPI) exclusion after gating for forward scatter (FSC) versus side scatter (SSC). EGFP detection allowed the identification and purification of N1ICD-overexpressing EGFP ${ }^{+}$cells. Specific $\mathrm{mAb}$ signals were defined against the background of irrelevant, isotypematched mAbs. Experiments were performed in a FACSCalibur flow cytometer (BD Biosciences) and analyzed with the FlowJo-v6.3.4 software package (Tree Star Inc., Ashland, OR, USA). Stained cells were purified by FACS, using a FACSAria cell sorter (BD Biosciences) under sterile conditions. The purity of the recovered cells was above $98 \%$.

In situ hybridization, hematoxylin-eosin, May GrümwaldGiemsa and immunofluorescence staining. Embryos were dissected and fixed with $4 \%$ paraformaldehyde in phosphate-buffered saline overnight at $4{ }^{\circ} \mathrm{C}$. Tissue embedding in paraffin, histological sectioning and hematoxylin and eosin staining were performed following standard protocols. In situ hybridization was carried out as described. ${ }^{58}$ Cytospin preparations of cell suspensions $\left(2.0-2.5 \times 10^{4}\right)$ were dried at room temperature and fixed in $100 \%$ methanol, before being stained with May Grümwald-Giemsa solution (Materlab S.L., Madrid, Spain). vWF immunostainings were performed on slides of cell suspensions recovered from MethoCult cultures, as described. ${ }^{57}$
Table 1 Antibodies used

\begin{tabular}{llll}
\hline Antibody & Conjugation & Clone & Company \\
\hline CD9 & Biotin & KMC8 & BD Biosciences \\
CD31 (PECAM-1) & PE/Biotin & MEC 13.3 & BD Biosciences \\
CD41 & PE & MWReg30 & BD Biosciences \\
CD45 & APC & $30-F 11$ & BD Biosciences \\
CD71 & PE & R17217 & e-Bioscience \\
C-Kit/CD117 & APC & $2 B 8$ & BD Biosciences \\
KDR/Flk1 & PE & Avas 12 $\alpha 1$ & BD Biosciences \\
F4/80 & APC & BM8 & e-Bioscience \\
Mac1/CD11b & PE & M1/70 & BD Biosciences \\
TER119 & Biotin/PerCP-Cy5.5 & TER-119 & BioLegend \\
Tie2/TEK & Biotin & TEK4 & e-Bioscience \\
Gr1 & Biotin & RB6-8C5 & BD Biosciences \\
Streptavidin & PE/APC/PE-Cy7 & & BioLegend \\
Anti-mouse vWF & Purified & Rabbit & Chemicon \\
Anti-mouse lgG & Purified & Rabbit & Chemicon \\
Anti-rabbit & Alexa-546 & Goat & Invitrogen \\
& & & \\
\hline
\end{tabular}

${ }^{a} A P C:$ Allophycocyanin

be-Bioscience, Hatfield, UK

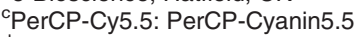

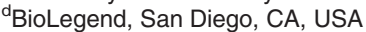

${ }^{\mathrm{e} C y} 7$ : Cyanin7

${ }^{f}$ Chemicon International Inc., Temecula, CA, USA

Confocal analysis. E9.5 WT and Tie2-Cre; N1ICD YSs were fixed $3 \mathrm{~h}$ in PFA 4\%. Nuclei were stained with DAPI 1:1000 (124653; Merck, Readington, NJ, USA) for 15' before microscopy scanning. YSs were scanned for EGFP expression with a Leica SPE confocal microscope (Leica Microsystems, Solms, Germany) and LasAF software (Leica Microsystems).

Clonal assays for progenitor identification. Purified EGFP ${ }^{+}$or WT YS cells $\left(0.3-3.0 \times 10^{3} / 50 \mu l\right)$ in DMEM (Lonza, Verviers, Belgium) were seeded in MethoCult M3434 methylcelullose medium (StemCell Technologies Inc., Vancouver, BC, Canada) and plated in triplicate in 48-well plates (Nunc, Thermo-Scientific, Roskilde, Denmark). Plates were kept at $37^{\circ} \mathrm{C}$ and $5 \% \mathrm{CO}_{2}$ for 7-10 days, and CFUs were counted by two independent observers under light microscopy (Leica-DFC 320 microscope, Leica Microsystems). Cell suspensions of the EGFP ${ }^{+}$CFUs were transferred to fibronectin-coated plates (BD Biosciences) and supplemented with the pro-endothelial factors basic fibroblast growth factor and vascular endothelial growth factor (Peprotech, London, UK), or used for cytospin preparations. For MK progenitor quantification (MK-CFU), viable CD41 ${ }^{+}$

cells were plated in IMDM (Lonza) at $0.1-3.0 \times 10^{3}$ in MegaCult-C medium (StemCell Technologies Inc.), in 2-well chamber slides and incubated at $37^{\circ} \mathrm{C}$ for 7-10 days. Slides were dried before staining with acetylthiocholiniodide (SigmaAldrich, St. Louis, MO, USA) as indicated by the manufacturer.

Proliferation, cell cycle and apoptosis analysis. Total YS cells were cultured over OPg stromal cells pretreated with mytomycin (Sigma-Aldrich) in IMDM supplemented with IL7 (Peprotech), IL3 (Stem Cell), SCF (Peprotech), FLT3 ligand (Peprotech) and GM-CSF (Peprotech). Cells were seeded in 96-well plates (Nunc, Rochester, NY, USA) and cultured for 7 days. The cells were recovered, counted and analyzed by FACS to quantify EGFP ${ }^{+}$cells. Aliquots of these cells were reseeded at $2 \times 10^{3} /$ well and recovered after 7 days. For cellcycle analysis, FACS-purified EGFP ${ }^{+}$or WT cells $\left(10^{5}\right)$ were fixed in pre-cooled $70 \%$ ethanol, treated with RNAse, stained for DNA with PI, and analyzed in a FACSCalibur flow cytometer (BD Biosciences). In other experiments, $10^{5}$ cells from WT or Tie2-Cre;N1ICD YS were incubated with Hoechst $33342(5 \mu \mathrm{g} / \mathrm{ml}$; Invitrogen, Carlsbad, CA, USA) and verapamil (20 $\mu \mathrm{M}$; Sigma-Aldrich) in IMDM at $37^{\circ} \mathrm{C}$ in the dark, and analyzed in a FACSVantage flow cytometer (BD Biosciences). Samples from adult thymus and a cell line were used as controls (data not shown). For in vivo S-phase cell analysis, pregnant females were intraperitoneally injected with $10 \mathrm{mM}(100 \mu \mathrm{l})$ 5-ethinyl-2'-deoxyuridine (EdU; Invitrogen), and EGFP ${ }^{+}$or WT cells were FACS purified. EdU incorporation was measured with the Click-iT EdU Flow Cytometry Kit (Invitrogen). Apoptotic cells were detected by flow cytometry using the 7-AAD Apoptosis Detection kit (Immunostep, Salamanca, Spain). 
Quantitative PCR. Total RNA from YS and FACS-purified cells were extracted with Trizol reagent (Invitrogen). cDNAs were synthesized using oligo(dT) as primer and avian myeloblastosis virus reverse transcriptase (Promega, Madison, WI, USA). PCR amplifications were performed with the FastStart PCR amplification kit (Roche Diagnostics, Mannheim, Germany) in a PM-200 DNA Engine Cycler (Bio-Rad Laboratories Ltd, Hercules, CA, USA) using specific primer pairs for transcripts of interest (Supplementary Table 2). Quantification of $\mathrm{G} \alpha \mathrm{S}$ expression was performed as described by quantitative real-time PCR (qRT-PCR) in a Light-Cycler 2.0 system, using the Light-Cycler FastStart DNA Master SYBR green I kit (Roche Diagnostics)..$^{59}$ The threshold cycle $\left(C_{T}\right)$ of each reaction was calculated using the LightCycler 4.0 software (Roche, Basel, Switzerland) and the relative amount of specific G $\alpha S$ cDNA in each sample was determined. Specific RT-PCRs were performed with equivalent amounts of $\mathrm{G} \alpha \mathrm{S}$ CDNA. PCR products were electrophoretically separated on $2 \%$ agarose gels and quantified from nonsaturated PCRs (at 25, 30 or 35 cycles) by using GelDoc-XR and Quantity One software (Bio-Rad Laboratories Ltd). The densitometry values were referred to those obtained for the $\mathrm{G} \alpha \mathrm{S}$ transcript. The ratio of gene expression of each transcript between EGFP ${ }^{+}$versus the mean of WT cells $(\Delta \Delta \mathrm{Ct}$ method) was calculated. To quantify primitive $(\beta \mathrm{H} 1)$ and definitive $(\beta 1)$ hemoglobins RT-qPCR was performed on a CFX96 Real-Time System using the SsoFast Supermix EvaGreen (Bio-Rad) with specific primer pairs (Supplementary Table 2). The Bio-Rad CFX Manager software was used to calculate the $C_{T}$ of each reaction.

Statistical analysis. Means and standard errors were calculated with GraphPad Prism 3.0 (GraphPad Software Inc., La Jolla, CA, USA) and P-values were obtained with the two-tailed Student's $t$-test.

\section{Conflict of Interest}

The authors declare no conflict of interest.

Acknowledgements. We are grateful to B Palacios, P Martínez and C Ruiz for husbandry, Dr. M Alía and C Prado for their technical cytometry assistance and Dr. N Serrano and Dr. B de Andrés for helpful discussions. We thank S Bartlett (CNIC) for English editing. IC is supported by a fellowship from the Ministerio de Economía y Competitividad (MINECO). PMR has been supported by a PhD fellowship (FPU program, MINECO) and by the Fundación Leticia Castillejo. This work was funded by grants SAF2007-65265, SAF2009-12596, SAF2012-33916, and ISCIII08/0685 (MINECO) and SAL-0304-2006 (Madrid Regional Government) to MLG; SAF200762445 and SAF2010-17555, RD12/0019/0003 and RD12/0042/0005 (MINECO) to JLdIP. The CNIC is supported by the MINECO and the Pro-CNIC Foundation.

\section{Author contributions}

MARM, MLG and JLP designed research; IC, PMR, LL and MSA performed research; IC and MLG contributed analytical tools; IC, PMR, MARM, MLG and JLP analyzed and interpreted data; IC and MLG performed statistical analysis; JLP, $M L G$, IC and PMR wrote the manuscript.

1. Artavanis-Tsakonas S, Rand MD, Lake RJ. Notch signaling: cell fate control and signal integration in development. Science 1999; 284: 770-776.

2. Radtke F, Fasnacht N, Macdonald HR. Notch signalling in the immune system. Immunity 2010; 32: 14-27.

3. Lieber T, Kidd S, Alcamo E, Corbin V, Young MW. Antineurogenic phenotypes induced by truncated Notch proteins indicate a role in signal transduction and may point to a novel function for Notch in nuclei. Genes Dev 1993; 7: 1949-1965

4. Rebay I, Fehon RG, Artavanis-Tsakonas S. Specific truncations of Drosophila Notch define dominant activated and dominant negative forms of the receptor. Cell 1993; 74: 319-329.

5. Struhl G, Fitzgerald K, Greenwald I. Intrinsic activity of the Lin-12 and Notch intracellular domains in vivo. Cell 1993; 74: 331-345.

6. Milner LA, Kopan R, Martin DI, Bernstein ID. A human homologue of the Drosophila developmental gene, Notch, is expressed in CD34 + hematopoietic precursors. Blood 1994; 83: 2057-2062.

7. Ellisen LW, Bird J, West DC, Soreng AL, Reynolds TC, Smith SD et al. TAN-1, the human homolog of the Drosophila notch gene, is broken by chromosomal translocations in T lymphoblastic neoplasms. Cell 1991; 66: 649-661.

8. Weng AP, Ferrando AA, Lee W, Morris JPt, Silverman LB, Sanchez-Irizarry C et al Activating mutations of NOTCH1 in human T cell acute lymphoblastic leukemia. Science 2004; 306: 269-271.
9. Jonsson Jl, Xiang Z, Pettersson M, Lardelli M, Nilsson G. Distinct and regulated expression of Notch receptors in hematopoietic lineages and during myeloid differentiation. Eur J Immunol 2001; 31: 3240-3247.

10. Singh N, Phillips RA, Iscove NN, Egan SE. Expression of notch receptors, notch ligands, and fringe genes in hematopoiesis. Exp Hematol 2000; 28: 527-534.

11. Walker L, Carlson A, Tan-Pertel HT, Weinmaster G, Gasson J. The notch receptor and its ligands are selectively expressed during hematopoietic development in the mouse. Stem Cells 2001; 19: 543-552.

12. Godin IE, Garcia-Porrero JA, Coutinho A, Dieterlen-Lievre F, Marcos MA. Para-aortic splanchnopleura from early mouse embryos contains B1a cell progenitors. Nature 1993; 364: $67-70$.

13. Marcos MA, Godin I, Cumano A, Morales S, García-Porrero JA, Dieterlen-Lievre F et al. Developmental events from hemopoietic stem cells to B-cell populations and Ig repertories. Immunol Rev 1994; 137: 155-171.

14. Silver L, Palis J. Initiation of murine embryonic erythropoiesis: a spatial analysis. Blood 1997; 89: 1154-1164.

15. Palis J, Robertson S, Kennedy M, Wall C, Keller G. Development of erythroid and myeloid progenitors in the yolk sac and embryo proper of the mouse. Development 1999; 126 : 5073-5084.

16. Jaffredo T, Nottingham W, Liddiard K, Bollerot K, Pouget C. de Bruijn M. From hemangioblast to hematopoietic stem cell: an endothelial connection? Exp Hematol 2005; 33: $1029-1040$.

17. Cumano A, Dieterlen-Lievre F, Godin I. Lymphoid potential, probed before circulation in mouse, is restricted to caudal intraembryonic splanchnopleura. Cell 1996; 86: 907-916.

18. Cumano A, Godin I. Pluripotent hematopoietic stem cell development during embryogenesis. Curr Opin Immunol 2001; 13: 166-171.

19. Medvinsky A, Dzierzak E. Definitive hematopoiesis is autonomously initiated by the AGM region. Cell 1996; 86: 897-906.

20. Muller AM, Medvinsky A, Strouboulis J, Grosveld F, Dzierzak E. Development of hematopoietic stem cell activity in the mouse embryo. Immunity 1994; 1: 291-301.

21. Orkin SH, Zon L. Hematopoiesis: an evolving paradigm for stem cell biology. Cell 2008; 132: 631-644.

22. Carlesso N, Aster JC, Sklar J, Scadden DT. Notch1-induced delay of human hematopoietic progenitor cell differentiation is associated with altered cell cycle kinetics. Blood 1999; 93: 838-848.

23. Karanu FN, Murdoch B, Gallacher L, Wu DM, Koremoto M, Sakano S et al. The notch ligand jagged-1 represents a novel growth factor of human hematopoietic stem cells. J Exp Med 2000; 192: 1365-1372.

24. Stier S, Cheng T, Dombkowski D, Carlesso N, Scadden DT. Notch1 activation increases hematopoietic stem cell self-renewal in vivo and favors lymphoid over myeloid lineage outcome. Blood 2002; 99: 2369-2378.

25. Varnum-Finney B, Xu L, Brashem-Stein C, Nourigat C, Flowers D, Bakkour S et al. Pluripotent, cytokine-dependent, hematopoietic stem cells are immortalized by constitutive Notch1 signaling. Nat Med 2000; 6: 1278-1281.

26. Kumano K, Chiba S, Kunisato A, Sata M, Saito T, Nakagami-Yamaguchi E et al. Notch1 but not Notch2 is essential for generating hematopoietic stem cells from endothelial cells. Immunity 2003; 18: 699-711.

27. Robert-Moreno A, Espinosa L, Sanchez MJ, de la Pompa JL, Bigas A. The notch pathway positively regulates programmed cell death during erythroid differentiation. Leukemia 2007; 21: 1496-1503.

28. Hamaguchi I, Huang XL, Takakura N, Tada J, Yamaguchi Y, Kodama $\mathrm{H}$ et al. In vitro hematopoietic and endothelial cell development from cells expressing TEK receptor in murine aorta-gonad-mesonephros region. Blood 1999; 93: 1549-1556.

29. Ema M, Yokomizo T, Wakamatsu A, Terunuma T, Yamamoto M, Takahashi S. Primitive erythropoiesis from mesodermal precursors expressing VE-cadherin, PECAM-1, Tie2, endoglin, and CD34 in the mouse embryo. Blood 2006; 108: 4018-4024.

30. Del Monte G, Grego-Bessa J, Gonzalez-Rajal A, Bolos V, De La Pompa JL. Monitoring Notch1 activity in development: Evidence for a feedback regulatory loop. Dev Dyn 2007; 236: 2594-2614.

31. Kisanuki YY, Hammer RE, Miyazaki J, Williams SC, Richardson JA, Yanagisawa M. Tie2Cre transgenic mice: a new model for endothelial cell-lineage analysis in vivo. Dev Biol 2001; 230: 230-242.

32. Murtaugh LC, Stanger BZ, Kwan KM, Melton DA. Notch signaling controls multiple steps of pancreatic differentiation. Proc Natl Acad Sci USA 2003; 100: 14920-14925.

33. Luna-Zurita L, Prados B, Grego-Bessa J, Luxan G, del Monte G, Benguria A et al. Integration of a Notch-dependent mesenchymal gene program and Bmp2-driven cell invasiveness regulates murine cardiac valve formation. J Clin Invest 2010; 120: 3493-3507.

34. Krebs LT, Shutter JR, Tanigaki K, Honjo T, Stark KL, Gridley T. Haploinsufficient lethality and formation of arteriovenous malformations in Notch pathway mutants. Genes Dev2004; 18: 2469-2473.

35. Robert-Moreno A, Espinosa L, de la Pompa JL, Bigas A. RBPjkappa-dependent Notch function regulates Gata2 and is essential for the formation of intra-embryonic hematopoietic cells. Development 2005; 132: 1117-1126.

36. Robert-Moreno A, Guiu J, Ruiz-Herguido C, Lopez ME, Ingles-Esteve J, Riera L et al. Impaired embryonic haematopoiesis yet normal arterial development in the absence of the Notch ligand Jagged1. EMBO J 2008; 27: 1886-1895. 
37. Yoon MJ, Koo BK, Song R, Jeong HW, Shin J, Kim YW et al. Mind bomb-1 is essential for intraembryonic hematopoiesis in the aortic endothelium and the subaortic patches. Mol Cell Biol 2008; 28: 4794-4804.

38. Lancrin C, Sroczynska P, Stephenson C, Allen T, Kouskoff V, Lacaud G. The haemangioblast generates haematopoietic cells through a haemogenic endothelium stage. Nature 2009; 457: 892-895.

39. Yamasaki S, Nobuhisa I, Ramadan A, Taga T. Identification of a yolk sac cell population with hematopoietic activity in view of CD45/c-Kit expression. Dev Growth Differ 2011; 53: 870-877.

40. Asari S, Sakamoto A, Okada S, Ohkubo Y, Arima M, Hatano M et al. Abnormal erythroid differentiation in neonatal bcl-6-deficient mice. Exp Hematol 2005; 33: 26-34.

41. McGrath KE, Frame JM, Fromm GJ, Koniski AD, Kingsley PD, Little J et al. A transient definitive erythroid lineage with unique regulation of the beta-globin locus in the mammalian embryo. Blood 2011; 117: 4600-4608.

42. Xu M, Matsuoka S, Yang F, Ebihara Y, Manabe A, Tanaka R et al. Evidence for the presence of murine primitive megakarycytopoiesis in the early yolk sac. Blood 2001; 97: 2016-2022.

43. Bertrand JT, Jalil A, Klaine M, Jung S, Cumano A, Godin I. Three pathways to mature macrophages in the early mouse yolk sac. Blood 2005; 106: 3004-3011.

44. Pimanda JE, Ottersbach K, Knezevic K, Kinston S, Chan WY, Wilson NK et al. Gata2, Fli1, and $\mathrm{Scl}$ form a recursively wired gene-regulatory circuit during early hematopoietic development. Proc Natl Acad Sci USA 2007; 104: 17692-17697.

45. Friedman AD. Transcriptional control of granulocyte and monocyte development. Oncogene 2007; 26: 6816-6828.

46. Alhashem YN, Vinjamur DS, Basu M, Klingmuller U, Gaensler KM, Lloyd JA. Transcription factors KLF1 and KLF2 positively regulate embryonic and fetal beta-globin genes through direct promoter binding. J Biol Chem 2011; 286: 24819-24827.

47. Medvinsky A, Rybtsov S, Taoudi S. Embryonic origin of the adult hematopoietic system: advances and questions. Development 2011; 138: 1017-1031.

48. Tang $Y$, Harrington A, Yang X, Friesel RE, Liaw L. The contribution of the Tie2 + lineage to primitive and definitive hematopoietic cells. Genesis 2010; 48: 563-567.
49. Takakura N, Huang XL, Naruse T, Hamaguchi I, Dumont DJ, Yancopoulos GD et al. Critical role of the TIE2 endothelial cell receptor in the development of definitive hematopoiesis. Immunity 1998; 9: 677-686.

50. Li Z, Chen MJ, Stacy T, Speck NA. Runx1 function in hematopoiesis is required in cells that express Tek. Blood 2006; 107: 106-110.

51. Oh P, Lobry C, Gao J, Tikhonova A, Loizou E, Manet J et al. In vivo mapping of notch pathway activity in normal and stress hematopoiesis. Cell Stem Cell 2013; 13 190-204.

52. Zeuner A, Francescangeli F, Signore M, Venneri MA, Pedini F, Felli N et al. The Notch2Jagged1 interaction mediates stem cell factor signaling in erythropoiesis. Cell Death Differ 2010; 18: 371-380.

53. Venkatesh DA, Park KS, Harrington A, Miceli-Libby L, Yoon JK, Liaw L. Cardiovascular and hematopoietic defects associated with Notch1 activation in embryonic Tie2-expressing populations. Circ Res 2008; 103: 423-431.

54. Poirault-Chassac S, Six E, Catelain C, Lavergne M, Villeval J-L, Vainchenker W et al. Notch/Delta4 signalling inhibits human megakaryocytic terminal differentiation. Blood 2010 116: 5670-5678.

55. Liu H, Chi AW, Arnett KL, Chiang MY, Xu L, Shestova $O$ et al. Notch dimerization is required for leukemogenesis and T-cell development. Genes Dev 2010; 24: 2395-2407.

56. Orford KW, Scadden DT. Deconstructing stem cell self-renewal: genetic insights into cell-cycle regulation. Nat Rev Genet 2008; 9: 115-128.

57. Serrano N, Cortegano I, Ruiz C, Alia M, de Andres B, Rejas MT et al. Megakaryocytes promote hepatoepithelial liver cell development in E11.5 mouse embryos by cell-to-cell contact and by vascular endothelial growth factor a signaling. Hepatology 2012; 56: 1934-1945.

58. de la Pompa JL, Wakeham A, Correira KM, Samper E, Brown S, Aguilera RJ et al. Conservation of the Notch signalling pathway in mammalian neurogenesis. Development 1997; 124: 1139-1148.

59. Gozalbo-Lopez B, Andrade P, Terrados G, de Andres B, Serrano N, Cortegano I et al. A role for DNA polymerase mu in the emerging DJH rearrangements of the postgastrulation mouse embryo. Mol Cell Biol 2009; 29: 1266-1275. 